Kai Cieliebak

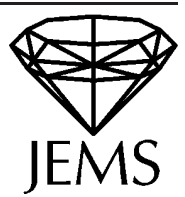

\title{
Handle attaching in symplectic homology and the Chord Conjecture
}

Received July 14, 2000 / final version received June 1, 2001

Published online August 1, 2001 - (c) Springer-Verlag \& EMS 2001

\begin{abstract}
Arnold conjectured that every Legendrian knot in the standard contact structure on the 3-sphere possesses a characteristic chord with respect to any contact form. I confirm this conjecture if the know has Thurston-Bennequin invariant -1 . More generally, existence of chords is proved for a standard Legendrian unknot on the boundary of a subcritical Stein manifold of any dimension. There is also a multiplicity result which implies in some situations existence of infinitely many chords.

The proof relies on the behaviour of symplectic homology under handle attaching. The main observation is that symplectic homology only changes in the presence of chords.
\end{abstract}

\section{Introduction}

\subsection{The Chord Conjecture}

In his 1986 paper [3], V.I. Arnold conjectured that every Legendrian knot in the standard contact structure on the 3 -sphere possesses a characteristic chord, i.e. an orbit of the Reeb vector field intersecting the knot at least twice.

The same question can be asked in the following more general situation: Let $N^{2 n-1}$ be a closed oriented manifold of dimension $2 n-1$. A contact form on $N$ is a 1 -form $\lambda$ such that $\lambda \wedge(d \lambda)^{n-1}$ is a positive volume form. The kernel $\xi=\operatorname{ker} \lambda$ of a contact form is called a (cooriented) contact structure, and the pair $(N, \xi)$ a contact manifold. A submanifold $L \subset(N, \xi)$ is called isotropic if it is tangent to $\xi$. An isotropic submanifold of the maximal possible dimension $n-1$ is called Legendrian.

A contact form determines a unique vector field $Y_{\lambda}$, called the Reeb vector field, by the conditions $i_{Y_{\lambda}} d \lambda=0$ and $\lambda\left(Y_{\lambda}\right)=1$. A characteristic chord for a contact form $\lambda$ and a Legendrian submanifold $L$ is an orbit of the Reeb vector field intersecting $L$ at least twice. Let us say that the (generalised) Chord Conjecture holds for a triple $(N, \lambda, L)$ if $L$ possesses a characteristic chord for $\lambda$, and that it holds for a triple $(N, \xi, L)$ if it holds for every triple $(N, \lambda, L)$ with $\xi=\operatorname{ker} \lambda$. Since Legendrian isotopies extend to ambient contact isotopies, if the Chord Conjecture

K. Cieliebak: Stanford University, Department of Mathematics, Stanford, CA 94305-2125, USA

Mathematics Subject Classification (2000): 53D, 57R, 58C 
holds for $(N, \xi, L)$ then it holds for $(N, \xi, \tilde{L})$ with any $\tilde{L}$ isotopic to $L$ (through Legendrian embeddings).

Note that the existence of a chord for a triple $(N, \lambda, L)$ can be checked directly from the Reeb flow of $\lambda$. The question becomes nontrivial if we fix the contact structure and allow the contact form and/or the Legendrian submanifold to vary. I am aware of only two nontrivial results concerning the Chord Conjecture: ${ }^{1}$

Theorem 1.1. (Ginzburg and Givental [17], [18]). The Chord Conjecture holds for $\left(\mathbb{R} P^{2 n-1}, \xi_{\text {st }}, L\right)$ where $\xi_{\text {st }}$ is the standard structure on $\mathbb{R} P^{2 n-1}=\left(\mathbb{C}^{n}\right)^{*} / \mathbb{R}^{*}$ and $L$ is isotopic to $\mathbb{R} P^{n-1}=\left(\mathbb{R}^{n}\right)^{*} / \mathbb{R}^{*} \subset \mathbb{R} P^{2 n-1}$. Moreover, the number of chords of lenght $\leq t$ grows at least linearly with $t$.

Theorem 1.2. (Abbas [1]). The Chord Conjecture holds for any triple $\left(S^{3}, \lambda, L\right)$ where $\lambda$ is a contact form induced by an embedding $S^{3} \hookrightarrow \mathbb{R}^{4}$ as a strictly convex hypersurface, and $L$ is a Legendrian knot with the following property: there exists an unknotted periodic Reeb orbit $P$ with self-linking number -1 such that $L$ and $P$ are not linked.

C. Abbas [2] is working on a variational approach to the Chord Conjecture on general tight 3-manifolds. Moreover, the symplectic field theory of Eliashberg, Givental and Hofer [12] is expected to provide another way to address the Chord Conjecture in any dimension. These approaches all rely on the study of holomorphic curves in symplectisations. In this paper, several cases of the Chord Conjecture are proved by a much simpler method involving holomorphic curves in Weinstein domains.

A Weinstein domain ([27], [11], [14]) is a quadruple $\left(W^{2 n}, \omega, X, \phi\right)$ where

- $(W, \omega)$ is a compact symplectic manifold with boundary;

- $X$ is an expanding vector field, i.e. $L_{X} \omega=\omega$;

- $\phi$ is a Morse function which has the boundary as a regular level set;

- the critical points of $\phi$ are nondegenerate zeroes of $X$, and $X \cdot \phi>0$ outside the critical points;

- $X$ is outward pointing along the boundary.

It follows that all critical points of $\phi$ have index $\leq n$. Call $\left(W^{2 n}, \omega, X, \phi\right)$ subcritical if all critical points of $\phi$ have index $<n$. Note that the 1 -form $\lambda:=i_{X} \omega$ satisfies $d \lambda=\omega$, and $\left.\lambda\right|_{\partial W}$ is a contact form.

A Weinstein homotopy is a smooth family $\left(W, \omega_{t}, X_{t}, \phi_{t}\right)_{t \in[0,1]}$ such that each $\left(W, \omega_{t}, X_{t}, \phi_{t}\right)$ has the properties of a Weinstein domain except that critical points of $\phi_{t}$ and zeroes of $X_{t}$ may be degenerate. In general, $\left(W, \omega_{0}\right)$ and $\left(W, \omega_{1}\right)$ will not be symplectomorphic. They will be symplectomorphic if the Weinstein homotopy is fixed near $\partial W$.

Weinstein domains arise naturally from Stein domains. These are triples $\left(W^{2 n}, J, \phi\right)$ where $(W, J)$ is a compact complex manifold of real dimension $2 n$,

1 After this paper was submitted, K. Mohnke [21] independently attacked the Chord Conjecture via Gromov's method of holomorphic disks. He proves existence of a chord for any compact Legendrian submanifold in the boundary of a subcritical Stein manifold, thus greatly generalizing Theorem 1.3 below. On the other hand, his method does not give multiplicity results as in Theorem 1.4. 
and $\phi: W \rightarrow \mathbb{R}$ is a strictly plurisubharmonic (i.e. $-d(d \phi \circ L)$ is a positive $(1,1)$ form) Morse function which is constant on $\partial W$ and has no critical points on $\partial W$. A Stein domain $\left(W^{2 n}, J, \phi\right)$ has an associated Weinstein structure $\left(\omega_{\phi}, X_{\phi}, \phi\right)$ where $\omega_{\phi}=-d(d \phi \circ J)$ and $i_{X_{\phi}} \omega_{\phi}=-d \phi \circ J$.

Another important example is the unit disk cotangent bundle $D T^{*} Q:=\{p \in$ $\left.T^{*} Q \mid\|p\| \leq 1\right\}$ with respect to a Riemannian metric on a compact manifold $Q^{n}$. $T^{*} Q$ carries a canonical $(\omega, X, \phi)$ which in local coordinates $\left(q_{j}, p_{j}\right)$ is given by $\omega=\sum d q_{j} \wedge d p_{j}, X=\sum p_{j} \frac{\partial}{\partial p_{j}}$, and $\phi=\sum p_{j}^{2}$. Note that $X$ and $\phi$ are degenerate along the zero section. But they can be perturbed (by adding a small Morse function on $Q$ to $\phi$ ) to a canonical Weinstein structure which is unique up to Weinstein homotopy.

It has been observed in [27] and [10] that the manifold $W \cup_{L} H_{k}^{2 n}$ obtained by attaching a $2 n$-dimensional $k$-handle with a standard framing to a Weinstein (respectively Stein) domain $W^{2 n}$ along an isotropic sphere $L^{k-1} \subset \partial W$ carries again a natural Weinstein (respectively Stein) structure (see Sect. 2 for definitions and precise statements). Call the handle subcritical if $k<n$.

The main result of this paper is the following:

Theorem 1.3. Let $\left(N^{2 n-1}, \xi\right), n \geq 2$, be the boundary of a subcritical Weinstein domain $W^{2 n}$, and $L \subset N$ a Legendrian sphere. Suppose that

$$
W \cup_{L} H_{n}^{2 n} \cup\{\text { subcritical handles }\}
$$

is Weinstein homotopic to

$$
D T^{*} Q \cup\{\text { subcritical handles }\}
$$

for some compact manifold $Q^{n}$. Then the Chord Conjecture holds for $(N, \xi, L)$.

This result can be improved to yield chords in specific homotopy classes. Denote by $\tilde{\pi}_{1}(W, L)$ the homotopy classes of maps $x:[0,1] \rightarrow W$ with $x(0), x(1) \in L$. They are in canonical one-to-one correspondence with free homotopy classes of loops in $W \cup_{L} H_{n}^{2 n}$, denoted by $\tilde{\pi}_{1}\left(W \cup_{L} H_{n}^{2 n}\right)$. Let

$$
M:=W \cup_{L} H_{n}^{2 n} \cup\{\text { subcritical handles }\} \cong D T^{*} Q \cup\{\text { subcritical handles }\}
$$

be the Weinstein domain in Theorem 1.3 , and denote by $i_{\#}: \tilde{\pi}_{1}(W, L) \rightarrow \tilde{\pi}_{1}(M)$, $j_{\#}: \tilde{\pi}_{1}(Q) \rightarrow \tilde{\pi}_{1}(M)$ the maps induced by the inclusions.

Theorem 1.4. Let $(N, \xi), L, W, M$ be as above. Then for every $\gamma \in \tilde{\pi}_{1}(Q)$ with $j_{\#} \gamma$ nontrivial there exists a chord $x$ for $(N, \xi, L)$ whose class $[x] \in \tilde{\pi}_{1}(W, L)$ satisfies $i_{\#}[x]=j_{\#} \gamma$.

In the remainder of this section I will describe examples where these theorems apply. Call an embedded Legendrian sphere $L^{n-1}$ in $\mathbb{R}^{2 n-1}$ with its standard contact form $d z-\sum_{j=1}^{n-1} y_{j} d x_{j}$ a standard Legendrian unknot if its (front) projection onto 
the $(x, z)$ subspace is a 2-valued graph over a ball with a standard cusp along its boundary, given by the formulae

$$
\begin{gathered}
z= \pm\left(1-\|x\|^{2}\right)^{\frac{3}{2}}, \quad\|x\| \leq 1 \\
y_{j}=\frac{\partial z}{\partial x_{j}}=\mp 3 \sqrt{1-\|x\|^{2}} x_{j} .
\end{gathered}
$$

(see [4] Chapter $\mathrm{X}$ for a picture of a flying saucer). Call an embedded Legendrian sphere $L^{n-1}$ in an arbitrary contact manifold $\left(N^{2 n-1}, \xi\right)$ a standard Legendrian unknot if it is Legendrian isotopic to a standard Legendrian unknot in a Darboux chart. Legendrian great circles in $S^{2 n-1}$ (images of $\mathbb{R}^{n} \cap S^{2 n-1} \subset \mathbb{C}^{n}$ under unitary maps) are examples of standard Legendrian unknots, and any two standard Legendrian unknots are Legendrian isotopic (Lemma 2.8 below).

Corollary 1.5. The Chord Conjecture holds for any triple $\left(N^{2 n-1}, \xi, L\right), n \geq 2$, where $(N, \xi)$ is the boundary of a subcritical Weinstein domain, and L is a standard Legendrian unknot.

This corollary follows from the fact that a standard Legendrian unknot can be disjoined from all the subcritical handles, and attaching a handle to the $2 n$-ball along a standard Legendrian unknot yields $T^{*} S^{n}$. The details are carried out in Sect. 2.4. As a special case we obtain

Corollary 1.6. The Chord Conjecture holds for $\left(S^{2 n-1}, \xi_{\mathrm{st}}, L\right)$ where L is isotopic to a Legendrian great circle.

In view of the covering $\left(S^{2 n-1}, \xi_{\mathrm{st}}, S^{n-1}\right) \rightarrow\left(\mathbb{R} P^{2 n-1}, \xi_{\mathrm{st}}, \mathbb{R} P^{n-1}\right)$, this implies the existence part (but not the linear growth) of Ginzburg/Givental's theorem.

For a physical example, consider a mechanical system defined by a potential $V: \mathbb{R}^{n} \rightarrow \mathbb{R}$ and Hamiltonian $H(q, p)=\frac{1}{2}|p|^{2}+V(q)$. Suppose that for some $E>\min V$

$$
\langle q, \nabla V(q)\rangle>2(V(q)-E) \text { for all } q \text { with } V(q) \leq E .
$$

For example, this condition is satisfied for very $E>\min V$ if $V$ has a unique minimum at the origin and all its level surfaces are star-shaped with respect to the origin. Condition $(V)$ implies that $\frac{1}{2} \sum_{i}\left(q_{i} d p_{i}-p_{i} d q_{i}\right)$ is a contact form on the level surface $\{H=E\} . L:=\{H=E\} \cap\{p=0\}$ is a standard Legendrian unknot, thus it possesses a characteristic chord by Corollary 1.6. In view of the time reversibility of the system, the chord has the following physical meaning:

Corollary 1.7. Suppose that $V$ satisfies condition $(V)$ for some $E>\min V$. Then there exists a libration motion of energy E, i.e. a motion that oscillates back and forth along the same orbit between two points on $\{V=E\}$.

This result, under weaker hypotheses, is due to Bolotin [5].

For $n=2$, the contact 3-manifolds filled by subcritical Stein manifolds are $S^{3}$ and $k$-fold connected sums $\#_{k}\left(S^{1} \times S^{2}\right)$. They all carry unique tight contact structures which are induced by the filling and will be denoted by $\xi_{\text {st }}$ [19]. The 
Thurston-Bennequin invariant $t b(L)$ of a null-homologous Legendrian knot $L$ in a contact 3-manifold is the linking number of $L$ with its push-off in the direction of the Reeb vector field. By the main result of [13], standard Legendrian unknots are precisely those Legendrian unknots with $t b=-1$. So Theorem 1.3 implies

Corollary 1.8. $(n=2)$. The Chord Conjecture holds for triples $\left(S^{3}, \xi_{\mathrm{st}}, L\right)$ and $\left(\#_{k}\left(S^{1} \times S^{2}\right), \xi_{\mathrm{st}}, L\right)$, where $L$ is a Legendrian unknot with $\operatorname{tb}(L)=-1$.

Finally, here are some explicit examples of nontrivial Legendrian knots:

Corollary 1.9. The Chord Conjecture holds for $\left(\#_{k}\left(S^{1} \times S^{2}\right), \xi_{\mathrm{st}}, L\right)$ where $L$ is a Legendrian knot running over the first $2 g \leq k$ handles with Kirby diagram [20] as in Fig. 1.
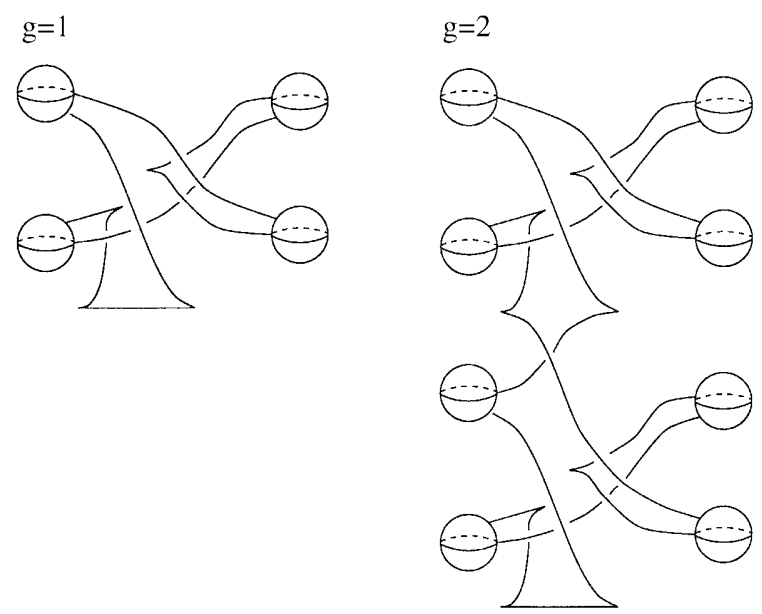

Fig. 1.

This corollary follows from the fact, proved in Sect. 2.4, that attaching a 2handle to $\tilde{\#}_{2 g}\left(S^{1} \times B^{3}\right)$ along this Legendrian knot $L$ yields the unit disk cotangent bundle $D T^{*} \Sigma_{g}$ of the surface of genus $g$. Combining this with Theorem 1.4 yields

Corollary 1.10. For $g>0$ the triple $\left(\#_{k}\left(S^{1} \times S^{2}\right), \xi_{\text {st }}, L\right)$ in Corollary 1.9 possesses infinitely many chords: at least one in each relative homotopy class of loops running over the first $2 \mathrm{~g}$ handles such that the corresponding free homotopy class on $\Sigma_{g}$ is nontrivial.

\subsection{Symplectic homology and proof of Theorems 1.3 and 1.4}

Symplectic homology has been introduced in the series of papers [15], [16], [7], [8]. In its most general form it associates to a symplectic manifold $(M, \omega)$ (satisfying some conditions), a free homotopy class of loops $\alpha \in \tilde{\pi}_{1}(M)$, a ring $R$, an integer $k$, and real parameters $a<b$ an $R$-module

$$
S H_{k}^{[a, b) ; \alpha}(M, \omega ; R)
$$


called the symplectic homology of $(M, \omega)$. It is invariant under symplectomorphisms and has certain functorial properties.

A suitable class of symplectic manifolds for the purpose of this paper are compact symplectic manifolds $\left(M^{2 n}, \omega\right)$ with boundary such that

$$
\int_{T^{2}} f^{*} \omega=0 \text { for every } f: T^{2} \rightarrow M
$$

$\partial M$ is $\omega$-convex.

The first hypothesis holds, for example, when $\omega$ is exact. Convexity means that there exists a 1 -form $\lambda$ on $\partial M$ such that $d \lambda=\left.\omega\right|_{\partial M}$ and $\lambda$ is a positive contact form, i.e. $\lambda \wedge(d \lambda)^{n-1}$ is a positive volume form with respect to the boundary orientation of $\partial M$. Note that a Weinstein domain satisfies $(\omega 1-2)$.

The coefficient ring is $\mathbb{Z}_{2}:=\mathbb{Z} / 2 \mathbb{Z}$, so that the symplectic homology modules are $\mathbb{Z}_{2}$ vector spaces. The interval $[a, b)$ is chosen as $(-\infty, \infty)$. If we ignore the grading $k$, symplectic homology associates to each compact symplectic manifold satisfying $(\omega 1-2)$ vector spaces

$$
S H_{*}^{\alpha}(M, \omega) \equiv S H_{*}^{(-\infty, \infty) ; \alpha}\left(M, \omega ; \mathbb{Z}_{2}\right)
$$

The construction is outlined in Sect. 3.1. Symplectic homology is invariant under symplectomorphisms and under homotopies of symplectic forms satisfying $(\omega 1-2)$ ([24], Lemma 3.7). In particular, the symplectic homology of Weinstein domains is invariant under Weinstein homotopies.

The proof of Theorems 1.3 and 1.4 is based on the behaviour of symplectic homology under handle attaching. For an embedding $i: W \hookrightarrow(M, \omega)$ and a free homotopy class $\alpha \in \tilde{\pi}_{1}(M)$ of loops on $M$ define

$$
\begin{gathered}
i_{\#}^{-1} \alpha:=\left\{\beta \in \tilde{\pi}_{1}(W) \mid i_{\#} \beta=\alpha\right\}, \\
S H_{*}^{i_{\#}^{-1} \alpha}(W, \omega):=\bigoplus_{\beta \in i_{\#}^{-1} \alpha} S H_{*}^{\beta}(W, \omega) .
\end{gathered}
$$

Theorem 1.11. Let $\left(W^{2 n}, \omega\right), n \geq 2$, be a compact symplectic manifold satisfying $(\omega 1-2)$. Let $(M, \omega)$ be the symplectic manifold obtained by attaching a $k$-handle to $W$ along an isotropic sphere $L^{k-1} \subset \partial W$ with a standard framing. Assume that $(M, \omega)$ satisfies $(\omega 1-2)$.

1. If $k<n$ then

$$
\operatorname{dim} S H_{*}^{\alpha}(M, \omega)=\operatorname{dim} S H_{*}^{i_{\#}^{-1} \alpha}(W, \omega) .
$$

2. If $k=n$ and $L$ possesses no chord in class $\alpha \in \tilde{\pi}_{1}(W, L) \cong \tilde{\pi}_{1}(M)$, and $\alpha$ is a nontrivial free homotopy class, then

$$
\operatorname{dim} S H_{*}^{\alpha}(M, \omega)=\operatorname{dim} S H_{*}^{i_{\#}^{-1} \alpha}(W, \omega) .
$$


3. If $k=n$ and $L$ possesses no chord in class $\alpha$, and $\alpha$ is the trivial free homotopy class, then either $\operatorname{dim} S H_{*}^{\alpha}(M, \omega)=\operatorname{dim} S H_{*}^{i_{\#}^{-1} \alpha}(W, \omega)=\infty$, or both dimensions are finite and

$$
\left|\operatorname{dim} S H_{*}^{\alpha}(M, \omega)-\operatorname{dim} S H_{*}^{i_{\#}^{-1} \alpha}(W, \omega)\right| \leq 1 .
$$

Now we are in the position to prove Theorems 1.3 and 1.4.

Proof of Theorem 1.4. Let $(N, \xi), L, W, M$ and $\gamma$ be as in the theorem. Assuming that $L$ possesses no chord in class $i_{\#}^{-1} j_{\#} \gamma$, we will compute the symplectic homology of $M$ in two different ways to get a contradiction.

First way: By assumption $W \cup_{L} H$ is Weinstein homotopic, up to adding and deleting handles of index $<n$, to a unit disk cotangent bundle $D T^{*} Q$ over a compact manifold $Q$. The symplectic homology of $D T^{*} Q$ has been computed by Viterbo [25] and Weber [26]: In class $\gamma$ it equals the singular homology of the component $\Lambda_{\gamma} Q \subset \Lambda Q$ of the free loop space,

$$
S H_{*}^{\gamma}\left(D T^{*} Q\right) \cong H_{*}\left(\Lambda_{\gamma} Q ; \mathbb{Z}_{2}\right) \neq\{0\} .
$$

By Theorem 1.11, adding subcritical handles does not change symplectic homology, so

$$
S H_{*}^{j_{\# \gamma}}(M) \neq\{0\} .
$$

Second way: Since $W$ is a subcritical Weinstein domain, repeated application of Theorem 1.11 shows $S H_{*}^{\beta}(W)=\{0\}$ for every $\beta$. If $L$ possesses no chord in class $i_{\#}^{-1} j_{\#} \gamma$ then, again by Theorem 1.11, $S H_{*}^{i_{*}^{-1} j_{\#} \gamma}\left(W \cup_{L} H_{n}^{2 n}\right)=\{0\}$. Here we use the hypothesis that $j_{\#} \gamma$ is nontrivial. Adding further subcritical handles does not affect symplectic homology, hence

$$
S H_{*}^{j \# \gamma}(M)=\{0\} .
$$

This contradiction concludes the proof.

Proof of Theorem 1.3. The proof is analogous to the proof of Theorem 1.4, computing the symplectic homology of $M$ in two different ways. Here we consider the total symplectic homology $S H_{*}^{*}(M):=\oplus_{\alpha} S H_{*}^{\alpha}(M)$.

First way: By a result of Sullivan [23], the homology $H_{*}\left(\Lambda Q ; \mathbb{Z}_{2}\right) \cong S H_{*}^{*}\left(D T^{*} Q\right)$ is infinite dimensional. By Theorem 1.11, adding and deleting a handle of index $<n$ changes the dimension of symplectic homology by at most 1 . Hence

$$
\operatorname{dim} S H_{*}^{*}(M)=\infty .
$$

Second way: By Theorem 1.11, if $L$ possesses no chord then adding a handle to $W$ along $L$ changes the dimension of symplectic homology by at most 1 . Hence

$$
\operatorname{dim} S H_{*}^{*}(M) \leq 1,
$$

and again we have a contradiction. 


\section{Attaching handles to Weinstein domains}

This section recollects some results on Weinstein domains that will be needed in the proof of the main theorem and its corollaries. Most of the results have counterparts in the category of Stein domains, but proofs are generally easier for Weinstein domains.

\subsection{Weinstein structures}

Recall the definition of a Weinstein domain $\left(W^{2 n}, \omega, X, \phi\right)$ from Sect. 1. A relative Weinstein domain satisfies all the conditions of a Weinstein domain except that $X$ may be inward pointing along some boundary components.

The symplectisation $N \times[a, b]$ of a contact manifold carries the canonical relative Weinstein structure (without critical points) $\omega:=d\left(e^{r} \lambda\right), X:=\frac{\partial}{\partial r}$, $\phi(y, r):=e^{r}$.

The proof of the following lemma is straighforward but somewhat lengthy and will be omitted.

Lemma 2.1. Let $\left(W, \omega, X_{0}, \phi_{0}\right)$ be a Weinstein domain, and $\Delta \subset W$ the union of all unstable manifolds for the flow of $-X_{0}$. Let $\left(\omega, X_{l o c}, \phi_{l o c}\right)$ be a Weinstein structure on a neighbourhood $W_{\text {loc }}$ of $\Delta$ having $\Delta$ as the union of the unstable manifolds.

Then there exists a Weinstein homotopy $\left(\omega, X_{t}, \phi_{t}\right)$ such that $\left(X_{t}, \phi_{t}\right)=$ $\left(X_{0}, \phi_{0}\right)$ outside $W_{\text {loc }}$ for all $t \in[0,1]$, and $\left(X_{1}, \phi_{1}\right)=\left(X_{\text {loc }}, \phi_{\text {loc }}\right)$ near $\Delta$.

Next we need a result about deformations of Weinstein structures. It is based on the following simple but useful lemma:

Lemma 2.2. Let $\left(\lambda_{t}\right)_{t \in[0,1]}$ be a smooth family of contact forms on a closed manifold $N^{2 n-1}$, independent of $t$ near 0 and 1 . Then there exists an $R>0$ and an increasing function $h:[0, R] \rightarrow[0,1]$ such that $h=0$ near $t=0, h=1$ near $t=1$, and $d\left(e^{r} \lambda_{h(r)}\right)$ is symplectic on $N \times[0, R], r \in[0, R]$.

Proof. The proof is a short computation:

$$
\begin{gathered}
\omega:=d\left(e^{r} \lambda_{h(r)}\right)=e^{r}\left[d r \wedge \lambda_{h}+d \lambda_{h}+h^{\prime}(r) d r \wedge \dot{\lambda}_{h}\right] \\
\omega^{n}=n e^{n r} d r \wedge\left(\lambda_{h}+h^{\prime}(r) \dot{\lambda}_{h}\right) \wedge(d \lambda)^{n-1} .
\end{gathered}
$$

So $\omega$ is symplectic iff $\left(\lambda_{h}+h^{\prime}(r) \dot{\lambda}_{h}\right)$ is positive on the Reeb vector field of $\lambda_{h}$, which is the case for $h^{\prime}(r)$ sufficiently small.

Consider two consecutive critical levels $a<b$ of $\phi$ in a Weinstein domain ( $W, \omega, X, \phi$ ) with unique critical points $p$ on level $a$ and $q$ on level $b$. Denote by $D_{p}^{+}=D_{p}^{+}(X)$ the stable manifold of $p$ with respect to $-X$, and by $D_{q}^{-}$the unstable manifold of $q$. Let $c$ be a regular level, $a<c<b$. 
Lemma 2.3. 1. If $D_{p}^{+} \cap D_{q}^{-}=\emptyset$, then the order of $p$ and $q$ can be reversed by a Weinstein homotopy. More precisely, there exists a family $\left(\phi_{t}\right)_{t \in[0,1]}$ such that $\left(\omega, X, \phi_{t}\right)$ are Weinstein structures for all $t, \phi_{0}=\phi, \phi_{t}=\phi$ outside a neighbourhood of $\{a \leq \phi \leq b\}$, and $\phi_{1}(p)>\phi_{1}(q)$.

2. Suppose there exists a family of isotropic spheres $L_{t} \subset\{\phi=c\}$ with $D_{q}^{-} \cap$ $\{\phi=c\}=L_{0}$. Then the Weinstein structure can be deformed such that $D_{q}^{-} \cap$ $\{\phi=c\}=L_{1}$. More precisely, there exists a family $\left(\omega_{t}, X_{t}\right)$ and constants $R_{t}$ such that $R_{0}=0,\left(\omega_{t}, X_{t}, \phi\right)$ are Weinstein structures for all $t,\left(\omega_{0}, X_{0}\right)=$ $(\omega, X),\left(\omega_{t}, X_{t}\right)=(\omega, X)$ on $\{\phi \leq a\},\left(\omega_{t}, X_{t}\right)=\left(e^{R_{t}} \omega, X\right)$ on $\{\phi \geq b\}$, and $D_{q}^{-}\left(X_{t}\right) \cap\{\phi=c\}=L_{t}$.

Proof. (1) Push up the level set $\{\phi=a\}$ along flow lines of $X$ until it intersects a neighbourhood of $q$ which is equivalent to the standard handle described in Subsect. 2.2. Then replace the level set by a level set above the critical point $q$ as described in [27].

(2) We will construct $\left(\omega_{1}, \phi_{1}\right)$. It will be clear from the construction that it can be done as a smooth family in $t$. Let $N:=\{\phi=c\}, \lambda:=\left.i_{X} \omega\right|_{N}$. Let $\psi_{t}: N \rightarrow N$ be a family of diffeomorphisms with $\psi_{1}=11, \psi_{t}\left(L_{0}\right)=L_{1-t}$ and $\psi_{t}^{*} \lambda=e^{f_{t}} \lambda$ for functions $f_{t}: N \rightarrow \mathbb{R}$. By Lemma 2.2, there exists a function $h$ such that $d\left(e^{r+f_{h(r)}} \lambda\right)$ is symplectic on $N \times[0, R]$. This form possesses the expanding vector field $\left(1+h^{\prime}(r) \dot{f}_{h(r)}\right)^{-1} \frac{\partial}{\partial r}$, hence $L_{0} \times[0, R]$ is Lagrangian and invariant. Now cut $W$ open along $N$ and glue in the relative Weinstein structure on $N \times[0, R]$ by $(x, r) \mapsto\left(\psi_{h(r)}(x), r\right)$. The glued in structure matches $(\omega, X, \phi)$ near $r=0$ and $\left(e^{R} \omega, X, \phi\right)$ near $r=R$. Moreover, $L_{0} \times[0, R]$ gets mapped to the invariant Lagrangian cylinder $\cup_{r \in[0, R]} L_{1-h(r)} \times\{r\}$, which agrees with $L_{1}$ near $r=0$ and $L_{0}$ near $r=R$.

\subsection{Handles}

Recall the contact surgery construction from [27]. Consider an isotropic submanifold $L^{k-1}$ of a contact manifold $\left(N^{2 n-1}, \xi\right)$. The form $\omega=d \lambda$ for some $\lambda$ with $\operatorname{ker} \lambda=\xi$ defines a natural conformal symplectic structure on $\xi$. Denote the $\omega$ orthogonal on $\xi$ by $\perp_{\omega}$. Since $L$ is isotropic, $T L \subset T L^{\perp_{\omega}}$. So the normal bundle of $L$ in $N$ is given by

$$
T N / T L=T N / \xi \oplus \xi /(T L)^{\perp_{\omega}} \oplus(T L)^{\perp_{\omega}} / T L .
$$

The Reeb vector field $Y_{\lambda}$ trivialises $T N / \xi$. The bundle $\xi /(T L)^{\perp_{\omega}}$ is canonically isomorphic to $T^{*} L$ via $v \mapsto i_{v} \omega$. The conformal symplectic normal bundle $\operatorname{CSN}(L):=(T L)^{\perp_{\omega}} / T L$ carries a natural conformal symplectic structure induced by $\omega$.

Now let $L^{k-1}$ be the standard sphere $S^{k-1}$. The embedding $S^{k-1} \subset \mathbb{R}^{k}$ provides a natural trivialisation of the bundle $\mathbb{R} \oplus T^{*} S^{k-1}$ where $\mathbb{R}$ denotes the trivial bundle. This trivialisation together with a conformally symplectic trivialisation of $\operatorname{CSN}\left(S^{k-1}\right)$ specifies a standard normal framing for $S^{k-1} \subset N$. Note that when $S^{k-1}$ is Legendrian then $\operatorname{CSN}\left(S^{k-1}\right)=\{0\}$, so there is a unique standard normal 
framing. Also note that for $S^{1} \subset N^{3}$ the standard normal framing differs from the trivialisation of the 2-dimensional bundle $\underline{\mathbb{R}} \oplus T^{*} S^{1}$ coming from a trivialisation of $T^{*} S^{1}$ by a single twist [19].

Proposition 2.4 (Weinstein [27]). Let $S^{k-1}$ be an isotropic sphere in a contact manifold $\left(N^{2 n-1}, \xi\right)$. Then the manifold $P:=\left(N^{2 n-1} \times[0,1]\right) \cup_{S^{k-1}} H_{k}^{2 n}$ obtained by attaching a $2 n$-dimensional $k$-handle $H_{k}^{2 n}$ to $[0,1] \times N^{2 n-1}$ along $S^{k-1} \times\{1\} \subset$ $N \times\{1\}$ with a standard framing carries a relative Weinstein structure $(\omega, X, \phi)$ such that

- $(\omega, X, \phi)$ is the standard structure on $N \times[0,1]$ as a symplectization;

- $X$ is inward pointing along $\partial^{-} P=N \times\{0\}$ and outward pointing along the other boundary component $\partial^{+} P$;

- $\phi$ has precisely one critical point $p$ of index $k$ in the interior of the handle whose unstable manifold intersects $N \times\{0\}$ in $S^{k-1} \times\{0\}$.

Thus $\partial^{+} P$ is a contact manifold resulting from surgery on $N$ along $S^{k-1}$. If $(N, \xi)$ is the boundary of a Weinstein domain $(W, \omega, X, \phi)$ then the proposition provides a Weinstein structure on $W \cup_{S^{k-1}} H_{k}^{2 n}$ extending the given one. The latter statement is also true in the Stein category [10].

Let us have a closer look at the standard handle $H_{k}^{2 n}$. Consider $\mathbb{R}^{2 n}$ with coordinates $\left(q_{1}, p_{1}, \ldots, q_{n}, p_{n}\right)$ and the Weinstein structure [27]

$$
\begin{aligned}
\omega & =\sum_{i=1}^{n} d q_{i} \wedge d p_{i}, \\
\phi & =\sum_{i=1}^{n-k}\left(\frac{1}{4} q_{i}^{2}+\frac{1}{4} p_{i}^{2}\right)+\sum_{i=n-k+1}^{n}\left(q_{i}^{2}-\frac{1}{2} p_{i}^{2}\right), \\
X & =\nabla \phi=\sum_{i=1}^{n-k}\left(\frac{1}{2} q_{i} \frac{\partial}{\partial q_{i}}+\frac{1}{2} p_{i} \frac{\partial}{\partial p_{i}}\right)+\sum_{i=n-k+1}^{n}\left(2 q_{i} \frac{\partial}{\partial q_{i}}-p_{i} \frac{\partial}{\partial p_{i}}\right) .
\end{aligned}
$$

Consider a function $\psi(x, y)$ of

$$
x:=\sum_{i=1}^{n} A_{i} q_{i}^{2}+\sum_{i=1}^{n-k} B_{i} p_{i}^{2}, \quad y:=\sum_{i=n-k+1}^{n} B_{i} p_{i}^{2}
$$

with coefficients $A_{i}, B_{i}>0$. It satisfies $X \cdot \psi>0$ provided that

$$
\frac{\partial \psi}{\partial x} \geq 0, \quad \frac{\partial \psi}{\partial y} \leq 0, \quad \frac{\partial \psi}{\partial x}(x, 0)>0, \quad \frac{\partial \psi}{\partial y}(0, y)<0,
$$

and the partial derivatives are not simultaneously zero. The handle is the set

$$
H_{k}^{2 n}:=\{\phi \geq-1\} \cap\{\psi \leq 1\}
$$

where the level surfaces $\{\phi=-1\}$ and $\{\psi=1\}$ agree outside a neighbourhood of the origin. The relative Weinstein structure on the handle is $(\omega, X, \psi)$. 
The Hamiltonian vector field of $\psi$ is given by

$$
X_{\psi}=\frac{\partial \psi}{\partial x}\left(\sum_{i=1}^{n} 2 A_{i} q_{i} \frac{\partial}{\partial p_{i}}+\sum_{i=1}^{n-k} 2 B_{i} p_{i} \frac{\partial}{\partial q_{i}}\right)+\frac{\partial \psi}{\partial y}\left(\sum_{i=n-k+1}^{n} 2 B_{i} p_{i} \frac{\partial}{\partial q_{i}}\right) .
$$

The function $f:=\sum_{i=n-k+1}^{n} q_{i} p_{i}$ satisfies $X_{\psi} \cdot f>0$ outside the critical points of $f$. This shows that all periodic orbits of $X_{\psi}$ are contained in $\left\{q_{n-k+1}=\right.$ $\left.p_{n-k+1}=\cdots=q_{n}=p_{n}=0\right\}$. The constants $A_{i}, B_{i}$ can be chosen so that none of the closed orbits has period 1 , except for the critical point at the origin. So we have shown

Lemma 2.5. The Weinstein structure $(\omega, X, \psi)$ on the handle can be chosen such that the only 1-periodic orbit on the handle is the critical point in its center.

Now suppose $k<n$. Then the core disk $D^{k}:=\left\{q_{1}=\cdots=q_{n}=p_{1}=\cdots=\right.$ $\left.p_{n-k}=0\right\} \cap H_{k}^{2 n}$ is contained in the $X$-invariant Legendrian handle

$$
H_{k}^{n}:=\left\{q_{1}=\cdots=q_{n}=0\right\} \cap H_{k}^{2 n} .
$$

The attaching sphere $S^{k-1}=D^{k} \cap\{\phi=-1\}$ is contained in the Legendrian submanifold $\partial^{-} H_{k}^{n}:=H_{k}^{n} \cap\{\phi=-1\}$. The Legendrian submanifold $\partial^{+} H_{k}^{n}:=$ $H_{k}^{n} \cap\{\psi=1\}$ is obtained from $\partial^{-} H_{k}^{n}$ by surgery along $S^{k-1}$ with cobordism $H_{k}^{n}$. In view of the normal form for isotropic setups [27], we can arrange that a given Legendrian submanifold $L \subset(N, \xi)$ intersects the handle in $\partial^{-} H_{k}^{n}$. So we have proved

Lemma 2.6. Let $L \subset(N, \xi)$ be a Legendrian submanifold containing the isotropic sphere $S^{k-1}, k<n$. Then the manifold $Q:=(L \times[0,1]) \cup_{S^{k-1}} H_{k}^{n}$ is $X$-invariant and Lagrangian. Its upper boundary $\partial^{+} Q$ is a Legendrian submanifold obtained from $\partial^{-} Q=L \times\{0\}$ by surgery along $S^{k-1}$.

\subsection{Standard Legendrian unknots}

The following lemma (which seems well-known, but I did not find a proof in the literature) states that the space of germs of contactomorphisms at a point is connected.

Lemma 2.7. Let $U$ be a neighbourhood of the origin in $\mathbb{R}^{2 n-1}$ with the standard contact structure. Then for every contact embedding $\phi: U \rightarrow \mathbb{R}^{2 n-1}$ with $\phi(0)=0$ there exists a neighbourhood $V \subset U$ of the origin and a smooth family of contact embeddings $\phi_{t}: V \rightarrow \mathbb{R}^{2 n-1}$ with $\phi_{t}(0)=0, \phi_{0}=\left.11\right|_{V}$ and $\phi_{1}=\left.\phi\right|_{V}$.

Proof. Let $X_{1}, y_{1}, \ldots, x_{n-1}, y_{n-1}, z$ be coordinates on $\mathbb{R}^{2 n-1}$. Denote by $\nabla_{x, y}$ and $\nabla_{x, y}^{2}$ the gradient and Hessian in the $(x, y)$ variables. Let $J_{0}$ be the standard complex structure on $\mathbb{R}^{2 n-2}$ and $\lambda=d z+\frac{1}{2} \sum_{j=1}^{n-1}\left(x_{j} d y_{j}-y_{j} d x_{j}\right)$ the standard contact form on $\mathbb{R}^{2 n-1}$. The first 2 steps of the proof are straightforward computations. 
Step 1: A contact embedding $\phi: U \rightarrow \mathbb{R}^{2 n-1}$ satisfies $\phi^{*} \lambda=\left(\phi^{*} f\right) \lambda$ for some function $f: U \rightarrow \mathbb{R}$. If $\phi(0)=0$ then its differential at the origin is of the form

$$
D \phi(0)=\left(\begin{array}{ll}
A & a \\
0 & \alpha
\end{array}\right)
$$

where $\alpha=f(0), a=-J_{0} \nabla_{x, y} f(0)$, and $A \in \mathbb{R}^{(2 n-2) \times(2 n-2)}$ satisfies $A^{*} d \lambda_{0}=$ $\alpha d \lambda_{0}$.

Step 2: Associated to a function $H: U \rightarrow \mathbb{R}$ is a contact vector field $X_{H}=H Y_{\lambda}+Z$, where $Y_{\lambda}=\frac{\partial}{\partial z}$ is the Reeb vector field and $Z \in \xi$ satisfies $\left.\left(i_{Z} d \lambda+d H\right)\right|_{\xi}=0$. If $H$ satisfies $H(0)=0, \nabla_{x, y} H(0)=0$, then the differential at the origin of $X_{H}$ is given by

$$
D X_{H}(0)=\left(\begin{array}{ll}
B & b \\
0 & \beta
\end{array}\right)
$$

where $\beta=\frac{\partial H}{\partial z}(0), b=-J_{0} \nabla_{x, y} \frac{\partial H}{\partial z}(0)$ and $B=J_{0} \nabla_{x, y}^{2} H(0)$.

Step 3: A contact embedding $\phi$ can be connected to a contact embedding $\psi$ with $D \psi(0)=11$.

Proof. The Lie algebra of the linear symplectic group $\operatorname{Sp}(2 n-2)$ consists of matrices $B=J_{0} S$ for symmetric matrices $S$. Since $S p(2 n-2)$ is connected, every symplectic matrix is a product of exponentials of matrices in the Lie algebra. This implies that $D \phi(0)$ as in Step 1 is a product of exponentials of $D X_{H}(0)$ as in Step 2 for suitable functions $H$.

The time-1 map $\phi_{H}$ of a contact vector field $X_{H}$ as in Step 2 is a contact embedding that is connected to the identity by the flow of $X_{H}$, and $D \phi_{H}(0)=$ $\exp \left[D X_{H}(0)\right]$. A suitable product of such time-1 maps will have the same differential at the origin as $\phi$, from which Step 3 follows.

Step 4: A contact embedding $\psi$ with $D \psi(0)=11$ can be connected to the identity.

Proof. Denote the components of $\psi$ corresponding to $(x, y, z)=\left(x_{j}, y_{j}, z\right)$ by $(\xi, \eta, \zeta)=\left(\xi_{j}, \eta_{j}, \zeta\right), j=1, \ldots, n-1$. Inspection of the 2 -jet of $\psi$ at the origin shows, as a consequence of $D \psi(0)=11$ and the contact condition, that all second order partial derivatives of $\zeta$ at the origin vanish, except possibly $\frac{\partial^{2} \zeta}{\partial z^{2}}$.

Since the rescaling $(x, y, z) \rightarrow\left(t x, t y, t^{2} z\right)$ is a contactomorphism for $t>0$, the maps

$$
\psi_{t}(x, y, z):=\left(\begin{array}{c}
\frac{1}{t} \xi\left(t x, t y, t^{2} z\right) \\
\frac{1}{t} \eta\left(t x, t y, t^{2} z\right) \\
\frac{1}{t^{2}} \zeta\left(t x, t y, t^{2} z\right)
\end{array}\right)
$$

define a family of contact embeddings for $0<t \leq 1$. Moreover, $D \psi(0)=\mathbb{1}$ and the vanishing of the second order partial derivatives of $\zeta$ imply that $\psi_{t} \rightarrow \mathbb{1}$ as $t \rightarrow 0$. 
Lemma 2.8. 1. Any two standard Legendrian unknots in a connected contact manifold are isotopic.

2. The standard great circle $S^{2 n-1} \cap \mathbb{R}^{n}$ is a standard Legendrian unknot in $S^{2 n-1}=\left\{Z \in \mathbb{C}^{n} \mid\|Z\|=1\right\}$ with its standard contact structure given by maximal complex subspaces.

Proof. 1. Suppose $L_{0}, L_{1}$ are standard Legendrian unknots in a connected contact manifold $(N, \xi)$. Let $\phi_{i}: \mathbb{R}^{2 n-1} \supset U_{i} \rightarrow N, i=0,1$, be Darboux charts such that $\phi_{i}^{-1}\left(L_{i}\right)$ are standard. After rescaling in the Darboux charts we may assume that $U_{1}=U_{2}=U$, and that $U$ contains the origin. Note that two standard Legendrian unknots in $\mathbb{R}^{2 n-1}$ are isotopic because their front projections can be deformed into one another through 2-valued graphs with standard cusps along the boundary of balls. Thus we may assume that $\phi_{0}^{-1}\left(L_{0}\right)=\phi_{1}^{-1}\left(L_{1}\right)$.

Since the group of contactomorphisms isotopic to the identity acts transitively on points of $N$, there exists a family of contact embeddings $\psi_{t}: U \rightarrow N$ such that $\psi_{0}=\phi_{0}$ and $\psi_{1}(0)=\phi_{1}(0)$. By Lemma 2.7 and possibly shrinking $U$ we can achieve $\psi_{1}=\phi_{1}$. Then $L_{t}:=\psi_{t} \circ \phi_{0}^{-1}\left(L_{0}\right)$ is the desired Legendrian isotopy.

2. Equip $\mathbb{R}^{2 n-1}$ with the contact form $\lambda:=d z-\sum_{j=1}^{n-1} y_{j} d x_{j}$. A standard Legendrian unknot is given by

$$
\begin{gathered}
z= \pm\left(1-\|x\|^{2}\right)^{\frac{3}{2}}, \quad\|x\| \leq 1 \\
y_{j}=\frac{\partial z}{\partial x_{j}}=\mp 3 \sqrt{1-\|x\|^{2}} x_{j} .
\end{gathered}
$$

Consider the Legendrian isotopy $f_{t}: D^{n-1}=\left\{x \mid\|x\|^{2} \leq 1\right\} \rightarrow \mathbb{R}^{2 n-1}$, $0<t \leq 1$,

$$
f_{t}(x):=\left(\frac{x}{t}, \mp 3 \sqrt{1-\|x\|^{2}} x, \pm \frac{\left(1-\|x\|^{2}\right)^{\frac{3}{2}}}{t}\right)
$$

which for $t=1$ is a parametrization of the standard unknot above. Consider also the embedding $\phi: \mathbb{R}^{2 n-1} \hookrightarrow S^{2 n-1}$ onto the open lower hemisphere given by $\phi:=\tilde{\phi} /\|\tilde{\phi}\|$,

$$
\tilde{\phi}(x, y, z):=\left(x+i y, \ldots, z-\frac{1}{2} \sum_{j=1}^{n-1} x_{j} y_{j}-2 i\right) .
$$

The embedding $\phi$ maps the contact structure $\{\lambda=0\}$ to the standard contact structure on $S^{2 n-1}$. Moreover,

$$
\begin{aligned}
\tilde{\phi} \circ f_{t}(x) & =\left(\frac{x}{t} \mp 3 \sqrt{1-\|x\|^{2}} i x, \frac{ \pm \sqrt{1-\|x\|^{2}}}{t}\left(1+\frac{\|x\|^{2}}{2}\right)-2 i\right), \\
\left\|\tilde{\phi} \circ f_{t}(x)\right\| & =\frac{1}{t^{2}}\left\{\|x\|^{2}+\left(1-\|x\|^{2}\right)\left(1+\frac{\|x\|^{2}}{2}\right)^{2}+O(t)\right\} .
\end{aligned}
$$


Thus $\phi \circ f_{t}$ converges as $t \rightarrow 0$ to the map

$$
x \mapsto \frac{\left(x, \pm \sqrt{1-\|x\|^{2}}\left(1+\frac{\|x\|^{2}}{2}\right)\right)}{\sqrt{\|x\|^{2}+\left(1-\|x\|^{2}\right)\left(1+\frac{\|x\|^{2}}{2}\right)^{2}}},
$$

which is a parametrization of the standard great circle in $S^{2 n-1}$.

\subsection{Proof of the corollaries assuming Theorem 1.3}

Corollary 1.5 follows immediately from Theorem 1.3 and the following

Proposition 2.9. Let $\left(W^{2 n}, \omega, X, \phi\right)$ be a subcritical Weinstein domain and $L^{n-1} \subset \partial W$ a standard Legendrian unknot with its canonical framing. Then the Weinstein domain $\left(W \cup_{L} H_{n}^{2 n}, \omega, X, \phi\right)$ is Weinstein homotopic to the unit cotangent bundle $D^{1} T^{*} S^{n}$ with subcritical handles attached.

Proof. By the second part of Lemma 2.3, we may replace $L$ by any Legendrian sphere isotopic to it in $\partial W$. So we can assume that $L$ is contained in an arbitrarily small Darboux chart, and that it does not intersect any stable manifold of the other critical points of $\phi$. Then by the first part of Lemma 2.3, we can push down the critical point on $H_{n}^{2 n}$ in $W \cup_{L} H_{n}^{2 n}$ to a level below all the other critical points.

This shows that $\left(W \cup_{L} H_{n}^{2 n}, \omega, X, \phi\right)$ is Weinstein homotopic to $\left(B^{2 n} \cup_{\tilde{L}} H_{n}^{2 n}\right.$, $\tilde{\omega}, \tilde{X}, \tilde{\phi})$ with subcritical handles attached, where $\tilde{\phi}$ has one critical point $p$ in the ball $B^{2 n}$ and one critical point $q$ on the handle $H_{n}^{2 n}$, and $\tilde{L} \subset \partial B^{2 n}$ is a standard Legendrian unknot.

After making $(\tilde{\omega}, \tilde{X}, \tilde{\phi})$ standard near $p$ and pushing down $\tilde{L}$ by the backward flow of $\tilde{X}$, we may assume that $(\tilde{\omega}, \tilde{X}, \tilde{\phi})$ are standard on $B^{2 n}$. Moreover, by Lemma 2.8 , we may assume that $\tilde{L}$ is the standard Legendrian great circle. Then the backward orbit of $\tilde{L}$ under the flow of $\tilde{X}$ is an embedded $n$-ball. Together with the core disk of the handle it forms a Lagrangian $n$-sphere $S^{n} \subset B^{2 n} \cup_{\tilde{L}} H_{n}^{2 n}$ which is the union of $p$ with the unstable manifold of $q$. By Lemma 2.1 we can achieve via another Weinstein homotopy that the Weinstein structure agrees with the standard structure on $T^{*} S^{n}$ near $S^{n}$. Then a neighbourhood of $S^{n}$ equals $D^{1} T^{*} S^{n}$, and ( $\left.W \cup_{L} H_{n}^{2 n}, \omega, X, \phi\right)$ is obtained (up to Weinstein homotopy) by attaching subcritical handles to this neighbourhood.

Corollary 1.9 is a direct consequence of Theorem 1.3 and the following

Proposition 2.10. Let $\left(\tilde{\#}_{k}\left(S^{1} \times B^{3}\right), \omega, X, \phi\right)$ be the 4-ball with $k$ 1-handles with its standard Weinstein structure. Let $L$ be a Legendrian knot in the boundary $\left(\#_{k}\left(S^{1} \times S^{2}\right), \xi_{\mathrm{st}}\right)$ running over the first $2 g \leq k$ handles with Kirby diagram as in 1 . Then the Weinstein domain obtained by attaching a 2-disk along $L$ is Weinstein homotopic to the unit cotangent bundle $D^{1} T^{*} \Sigma$ of a surface of genus $g$ with $(k-2 g)$ 1-handles attached. 
Proof. In the Kirby diagram 1, remove the parts of the knot $L$ running over the handle and at every 3-ball connect the two strands entering a handle by a cusp. The resulting knot $\tilde{L}$ is easily seen to be Legendrian isotopic to the standard unknot in $S^{3}=\partial B^{4}$. Thus we can choose the Weinstein structure $(\omega, \tilde{X}, \tilde{\phi})$ on $B^{4}$ such that the backward orbit of $\tilde{L}$ under $\tilde{X}$ is an embedded 2-disk containing the critical point of $\tilde{\phi}$. The knot $L$ is obtained from $\tilde{L}$ by a sequence of 0 -surgeries on the handles. So by Lemma 2.6, $L$ bounds an $X$-invariant Lagrangian surface in the resulting 4-manifold $M^{4}$ containing all the critical points of the extended function $\phi$. Attaching a 2-handle to $M$ along $L$ caps off this surface to a closed invariant Lagrangian surface $\Sigma$ of genus $g$ in $M \cup_{L} H_{2}^{4}$. Thus $\Sigma$ is the union of all unstable manifolds, and $M \cup_{L} H_{2}^{4}$ retracts onto $\Sigma$ under the backward flow of $X$. In view of the Lemma 2.1, this proves that $M \cup_{L} H_{2}^{4}$ is Weinstein homotopic to $T^{*} \Sigma$ with its standard structure.

\section{Symplectic homology and handle attaching}

\subsection{Symplectic homology}

Let us first define the appropriate version of symplectic homology, essentially following the lines of [6]. For details see [15], [7].

Throughout this section, $\left(M^{2 n}, \omega\right)$ is a compact symplectic manifold with boundary such that

$$
\int_{T^{2}} f^{*} \omega=0 \text { for every } f: T^{2} \rightarrow M
$$

$\partial M$ is $\omega$-convex.

The first hypothesis holds, for example, when $\omega$ is exact. Convexity means that there exists a 1 -form $\lambda$ on $\partial M$ such that $d \lambda=\left.\omega\right|_{\partial M}$ and $\lambda$ is a positive contact form, i.e. $\lambda \wedge(d \lambda)^{n-1}$ is a positive volume form with respect to the boundary orientation of $\partial M$. Let $Y_{\lambda}$ be the Reeb vector field and $\xi$ the contact structure defined by $\lambda$. Define the action spectrum of the boundary

$$
\mathcal{A}(\partial M, \lambda):=\left\{\int_{y} \lambda \mid y \text { closed orbit of } Y_{\lambda}\right\} .
$$

In coordinates $(y, r)$ on a collar neighbourhood $\partial M \times(-\delta, 0]$ we have $\omega=d\left(e^{r} \lambda\right)$. Define the completion $(\hat{M}, \hat{\omega})$ by

$$
\begin{gathered}
\hat{M}:=M \cup_{\partial M}(\partial M \times[0, \infty)), \\
\hat{\omega}:=d \hat{\lambda}, \hat{\lambda}:=e^{r} \lambda \text { on } \partial M \times[0, \infty) .
\end{gathered}
$$

A 1-periodic time-dependent Hamiltonian $H: S^{1} \times \hat{M} \rightarrow \mathbb{R}$ is called admissible if

$$
H(t,(y, r))=a e^{r}+b \quad \text { on } \partial M \times[0, \infty)
$$


where $a>0, b$ are constants and $a \notin \mathcal{A}(\partial M, \lambda)$. Define the Hamiltonian vector field $X_{H}$ by

$$
d_{x} H(t, x)=-\omega\left(X_{H}(t, x), \cdot\right) .
$$

Note that $X_{H}(t,(y, r))=a Y_{\lambda}(y)$ on $\partial M \times[0, \infty)$, so for $a \notin \mathcal{A}(\partial M, \lambda)$ all 1periodic solutions of the Hamiltonian system $\dot{x}=X_{H}(t, x)$ are contained in $M$. Call $H$ regular if all 1-periodic solutions are nondegenerate, i.e. the linearized Hamiltonian flow along $x$ does not have 1-periodic solutions.

Fix a reference loop $x_{\alpha}$ in each free homotopy class $\alpha$ of loops in $\hat{M}$ and arbitrarily assign it an action $A\left(x_{\alpha}\right)$. For a smooth loop $x: S^{1} \rightarrow \hat{M}$ with $[x]=\alpha$ define its Hamiltonian action as

$$
A_{H}(x):=\int_{[0,1] \times S^{1}} \bar{x}^{*} \hat{\omega}-\int_{0}^{1} H(t, x(t)) d t+A\left(x_{\alpha}\right),
$$

where $\bar{x}:[0,1] \times S^{1} \rightarrow \hat{M}$ satisfies $\bar{x}(0, t)=x_{\alpha}(t), \bar{x}(1, t)=x(t)$. The action does not depend on the choice of $\bar{x}$ because the integral of $\hat{\omega}$ vanishes over tori. Critical points of $A_{H}$ are precisely the 1-periodic solutions of $X_{H}$.

Fix a trivialisation of $T \hat{M}$ over each reference loop $x_{\alpha}$. For a 1-periodic orbit $x$ and an extension $\bar{x}$ as above let $\operatorname{ind}_{C Z}(x, \bar{x})$ be the Conley-Zehnder index ([9], [22]) of $x$ in the trivialisation of $\left.T \hat{M}\right|_{x}$ which extends over $[0,1] \times S^{1}$ to match the trivialisation of $\left.T \hat{M}\right|_{x_{\alpha}}$. Let

$$
\begin{aligned}
\mathcal{P}^{\alpha}(H):= & \left\{(x, \bar{x}) \mid x: S^{1} \rightarrow \hat{M}, \dot{x}=X_{H}(t, x),[x]=\alpha,\right. \\
& \left.\bar{x}:[0,1] \times S^{1} \rightarrow \hat{M}, \bar{x}(0, \cdot)=x_{\alpha}, \bar{x}(1, \cdot)=x\right\} / \sim,
\end{aligned}
$$

where $(x, \bar{x}) \sim(y, \bar{y})$ iff $x=y$ and $\operatorname{ind}_{C Z}(x, \bar{x})=\operatorname{ind}_{C Z}(y, \bar{y})$. Each equivalence class $[x, \bar{x}]$ possesses a well-defined action $A_{H}([x, \bar{x}])=A_{H}(x)$ and ConleyZehnder index. Let

$$
\mathcal{P}_{k}^{\alpha}(H):=\left\{[x, \bar{x}] \in \mathcal{P}^{\alpha}(H) \mid \operatorname{ind}_{C Z}([x, \bar{x}])=k\right\}
$$

Note that for every 1-periodic solution $x$ there is at most one element $[x, \bar{x}]$ in $\mathcal{P}_{k}^{\alpha}(H)$, hence for regular admissible $H$ the set $\cup_{\alpha} \mathcal{P}_{k}^{\alpha}(H)$ is finite for every $k$.

A Hamiltonian $H: \mathbb{R} \times S^{1} \times \hat{M} \rightarrow \mathbb{R}$ is called a monotone homotopy between admissible Hamiltonians $H_{-}, H_{+}$if

$$
\begin{gathered}
\frac{\partial H}{\partial s}(s, t, x) \leq 0 \quad \text { for all }(s, t, x), \\
H(s, t, x)=\left\{\begin{array}{l}
H_{-}(t, x) \quad \text { for } s \leq-s_{0} \\
H_{+}(t, x) \quad \text { for } s \geq s_{0},
\end{array}\right. \\
H(s, t,(y, r))=a(s) e^{r}+b(s) \quad \text { on } \partial M \times[0, \infty), \\
a^{\prime}(s)<0 \text { for } s \in\left(-s_{0}, s_{0}\right) .
\end{gathered}
$$


Pick an $(s, t)$-dependent almost complex structure $J(s, t, x)$ on $\hat{M}$ such that

$$
\begin{gathered}
\hat{\omega}(\cdot, J(s, t, x) \cdot) \text { is a Riemannian metric for every }(s, t), \\
J(s, t, x)= \begin{cases}J_{-}(t, x) & \text { for } s \leq-s_{0} \\
J_{+}(t, x) & \text { for } s \geq s_{0},\end{cases} \\
J(s, t,(y, r)): \begin{cases}\xi \rightarrow \xi & \text { on } \partial M \times[0, \infty) . \\
\frac{\partial}{\partial r} \rightarrow Y_{\lambda} & \\
Y_{\lambda} \rightarrow-\frac{\partial}{\partial r} & \end{cases}
\end{gathered}
$$

Then the $L^{2}$ gradient of $A_{H(s)}$ (for fixed $s$ ) at $s: S^{1} \rightarrow \hat{M}$ is given by

$$
A_{H(s)}^{\prime}(x)=-J(s, t, x) \dot{x}-\nabla_{J(s, t)} H(s, t, x),
$$

where $\nabla_{J(s, t)}$ is the gradient with respect to the metric $\hat{\omega}(\cdot, J(s, t, x) \cdot)$. A smooth map $u: \mathbb{R} \times S^{1} \rightarrow \hat{M}$ is a gradient trajectory for $A_{H}$ connecting periodic orbits $\left[x_{-}, \bar{x}_{-}\right] \in \mathcal{P}^{\alpha}\left(H_{-}\right),\left[x_{+}, \bar{x}_{+}\right] \in \mathcal{P}^{\alpha}\left(H_{+}\right)$if

$$
\begin{gathered}
\frac{\partial u}{\partial s}+J(s, t, u) \frac{\partial u}{\partial t}+\nabla_{J(s, t)} H(s, t, u)=0, \\
u(s, \cdot) \longrightarrow x_{ \pm} \text {in } C^{\infty} \text { as } s \rightarrow \pm \infty \\
{\left[x_{+}, \bar{x}_{-} \# u\right]=\left[x_{+}, \bar{x}_{+}\right],}
\end{gathered}
$$

where $\bar{x}_{-} \# u$ denotes the concatenation along $x_{-}$. Note that the action $A_{H(s)}(u(s, \cdot))$ is increasing in $s$. For generic $J$ the spaces

$$
\mathcal{M}\left(\left[x_{1}, \bar{x}_{1}\right],\left[x_{2}, \bar{x}_{2}\right] ; H, J\right)
$$

of solutions of (u1-3) are finite dimensional manifolds of dimension ind ${ }_{C Z}\left(\left[x_{+}, \bar{x}_{+}\right]\right)$ $-\operatorname{ind}_{C Z}\left(\left[x_{-}, \bar{x}_{-}\right]\right)$.

For regular admissible $H=H(t, x)$ let $C_{k}^{\alpha}(H)$ be the finite dimensional vector space over $\mathbb{Z}_{2}=\mathbb{Z} / 2 \mathbb{Z}$ with basis $\mathcal{P}_{k}^{\alpha}(H)$. Define linear maps

$$
\begin{aligned}
\partial_{k} & =\partial_{k}(H, J): C_{k}^{\alpha}(H) \rightarrow C_{k-1}^{\alpha}(H), \\
\partial_{k}[x, \bar{x}] & :=\sum_{[y, \bar{y}] \in P_{k-1}^{\alpha}(H)}\langle[x, \bar{x}],[y, \bar{y}]\rangle_{1} \cdot[y, \bar{y}],
\end{aligned}
$$

where $\langle[x, \bar{x}],[y, \bar{y}]\rangle_{1}$ is the (finite!) number $\bmod 2$ of 1 -dimensional components of $\mathcal{M}([y, \bar{y}],[x, \bar{x}] ; H, J)$. These maps satisfy $\partial_{k-1} \circ \partial_{k}=0$. The homology

$$
F H_{k}^{\alpha}(H):=\operatorname{ker}\left(\partial_{k}\right) / \operatorname{im}\left(\partial_{k+1}\right)
$$

is called the Floer homology of $H$. The Floer homology is independent of $J$, but it does depend on $H$. Introduce a partial ordering on the set of admissible Hamiltonians via

$$
H_{1} \leq H_{2}: \Longleftrightarrow H_{1}(t, x) \leq H_{2}(t, x) \text { for all }(t, x) .
$$


A monotone homotopy $H(s, t, x)$ between two regular Hamiltonians $H_{1} \leq H_{2}$ (going from $H_{2}$ to $H_{1}$ ) induces a linear map

$$
\begin{gathered}
\sigma_{k}(H, J): C_{k}^{\alpha}\left(H_{1}, J_{1}\right) \rightarrow C_{k}^{\alpha}\left(H_{2}, J_{2}\right), \\
\sigma_{k}(H, J)\left[x_{1}, \bar{x}_{1}\right]:=\sum_{\left[x_{2}, \bar{x}_{2}\right] \in \mathcal{P}_{k}^{\alpha}\left(H_{2}\right)}\left\langle\left[x_{1}, \bar{x}_{1}\right],\left[x_{2}, \bar{x}_{2}\right]\right\rangle_{0} \cdot\left[x_{2}, \bar{x}_{2}\right],
\end{gathered}
$$

where $\left\langle\left[x_{1}, \bar{x}_{1}\right],\left[x_{2}, \bar{x}_{2}\right]\right\rangle_{0}$ denotes the (finite) number mod 2 of 0 -dimensional components of $\mathcal{M}\left(\left[x_{2}, \bar{x}_{2}\right],\left[x_{1}, \bar{x}_{1}\right] ; H, J\right)$. It turns out that $\sigma_{k}(H, J)$ is a chain map. The induced map on Floer homology does not depend on the choice of $(H, J)$. Denote it by

$$
\sigma_{k}\left(H_{1}, H_{2}\right): F H_{k}^{\alpha}\left(H_{1}\right) \rightarrow F H_{k}^{\alpha}\left(H_{2}\right)
$$

For 3 regular Hamiltonians $H_{1} \leq H_{2} \leq H_{3}$ the corresponding maps satisfy the composition law

$$
\sigma_{k}\left(H_{2}, H_{3}\right) \circ \sigma_{k}\left(H_{1}, H_{2}\right)=\sigma_{k}\left(H_{1}, H_{3}\right) \text {. }
$$

Define the symplectic homology

$$
S H_{k}^{\alpha}(M, \omega):=\underset{\lim }{\longrightarrow} H_{k}^{\alpha}(H),
$$

where the direct limit is taken over admissible Hamiltonians tending to $+\infty$ with respect to the partial ordering $\leq$.

A free homotopy class $\alpha$ determines a component $\Lambda_{\alpha}$ of the free loop space. Fix a reference loop $x_{\alpha}$ in each $\Lambda_{\alpha}$. Evaluation of $c_{1}(T M)$ on tori defines a homomorphism $\pi_{1}\left(\Lambda_{\alpha} ; x_{\alpha}\right) \rightarrow \mathbb{Z}$. Its image is either 0 , in which case we set $c_{\alpha}:=\infty$, or $c_{\alpha} \cdot \mathbb{Z}$ for some $c_{\alpha} \in \mathbb{N}$. For $c_{\alpha}<\infty$ symplectic homology is $2 c_{\alpha}$-periodic,

$$
S H_{k+2 c_{\alpha}}^{\alpha}(M, \omega)=S H_{k}^{\alpha}(M, \omega) \text {. }
$$

Define

$$
\operatorname{dim} S H_{*}^{\alpha}(M, \omega):= \begin{cases}\sum_{k=0}^{2 c_{\alpha}-1} \operatorname{dim} S H_{k}^{\alpha}(M, \omega) & \text { if } c_{\alpha}<\infty \\ \sum_{k=-\infty}^{\infty} \operatorname{dim} S H_{k}^{\alpha}(M, \omega) & \text { if } c_{\alpha}=\infty\end{cases}
$$

Remark 3.1. It follows from the definition that $S H_{k}^{\alpha}(M, \omega)$ can be computed using any cofinal sequence $H_{1} \leq H_{2} \leq \ldots$ of admissible Hamiltonians. Moreover, if $\omega_{i}$ is a sequence of symplectic forms with $\omega_{i}=\omega$ near $\partial M$, then $S H_{k}^{\alpha}(M, \omega)$ can be computed as the direct limit of $H F_{k}^{\alpha}\left(\omega_{i} ; H_{i}\right)$ with connecting homomorphisms $\sigma\left(\omega_{i}, H_{i} ; \omega_{j}, H_{j}\right)$ defined by counting gradient trajectories with an $s$-dependent symplectic form connecting $\omega_{i}$ and $\omega_{j}$ (see [24] for details). 


\subsection{The Annulus Lemma}

Extend the function $r$ from $\partial M \times[0, \infty)$ to $\hat{M}$ by setting $r:=0$ on $M$. For $x: S^{1} \rightarrow \hat{M}$ write $r(t)=r \circ x(t)$. For $R \geq 1$ consider the "annulus"

$$
Z(R):=\left\{x \in C^{\infty}\left(S^{1}, \hat{M}\right) \mid \max _{t \in S^{1}} r(t) \geq 2 R, \min _{t \in S^{1}} r(t) \leq 3 R\right\} .
$$

The Annulus Lemma states that for a gradient trajectory $u$ to traverse $Z(R)$ it needs arbitrarily large energy. It is based on the following estimate:

Lemma 3.2. Let $H$ be an admissible Hamiltonian $H(t, x)$ or a monotone homotopy $H(s, t, x)$. Then there exists an $\varepsilon>0$ such that for every $R \geq 1, s \in \mathbb{R}$ and $x \in Z(R)$,

$$
-\int_{0}^{1} \frac{\partial H}{\partial s}(s, t, x) d t+\left\|A_{H(s)}^{\prime}(x)\right\|_{L^{2}(d t)}^{2} \geq \varepsilon\left\|A_{H(s)}^{\prime}(x)\right\|_{L^{2}(d t)} .
$$

Proof. Denote by ||$_{r}$ the metric induced by $\hat{\omega}$ on $\partial M \times[0, \infty)$ and by || the metric induced on $\partial M$. On $\partial M \times[0, \infty)$ we have

$$
\begin{gathered}
\dot{x}-X_{H}(t, x)=\dot{r} \frac{\partial}{\partial r}+\left(\dot{y}-a(s) Y_{\lambda}(y)\right), \\
\left|\frac{\partial}{\partial r}\right|_{r}^{2}=\left|Y_{\lambda}\right|_{r}^{2}=e^{r}, \quad|v|_{r}^{2}=e^{r}|v|^{2} \text { for } v \in \xi .
\end{gathered}
$$

We will treat the case when $H$ is a monotone homotopy, the $s$-independent case being similar but simpler. By assumption, $H=a(s) e^{r}+b(s)$ on $\partial M \times[0, \infty)$, $a(s)=a_{1}$ for $s \leq-s_{0}, a(s)=a_{2}$ for $s \geq s_{0}, a_{1}, a_{2} \notin \mathcal{A}(\partial M, \lambda)$ and $a^{\prime}(s)<0$ for $s \in\left(-s_{0}, s_{0}\right)$. Hence there exist $\varepsilon>0$ and $\tilde{s}_{0}<s_{0}$ such that

$$
\begin{gathered}
\left\|\dot{y}-a(s) Y_{\lambda}(y)\right\|_{L^{2}(d t)} \geq \varepsilon \text { for all }|s| \geq \tilde{s}_{0}, y \in C^{\infty}\left(S^{1}, \partial M\right) ; \\
a^{\prime}(s) \leq-\varepsilon^{2} \text { for }|s| \leq \tilde{s}_{0} .
\end{gathered}
$$

For $R \geq 1$ and $x \in Z(R)$ we distinguish two cases.

Case 1: There exist $t_{1}<t_{2}$ such that $\left|r\left(t_{2}\right)-r\left(t_{1}\right)\right| \geq R$.

In this case,

$$
\begin{aligned}
\left\|A_{H(s)}^{\prime}(x)\right\|_{L^{2}(d t)}^{2} & \geq \int_{t_{1}}^{t_{2}}|\dot{r}|^{2} e^{r} d t \\
& \geq\left(\int_{t_{1}}^{t_{2}}|\dot{r}| d t\right)^{2} \\
& \geq R^{2} \\
& \geq \varepsilon^{2} \text { for } \varepsilon \leq 1 .
\end{aligned}
$$

Case 2: $R \leq r(t) \leq 4 R$ for all $t \in S^{1}$.

In this case either $\left\|A_{H(s)}^{\prime}(x)\right\|_{L^{2}(d t)} \geq \varepsilon$ and we are done; or $\left\|A_{H(s)}^{\prime}(x)\right\|_{L^{2}(d t)}<\varepsilon$, which implies $|s| \leq \tilde{s}_{0}$, hence $a^{\prime}(s) \leq-\varepsilon^{2}$ and

$$
-\int_{0}^{1} \frac{\partial H}{\partial s}(s, t, x) d t \geq \varepsilon^{2} \geq \varepsilon\left\|A_{H(s)}^{\prime}(x)\right\|_{L^{2}(d t)} .
$$


Lemma 3.3 (Annulus Lemma). Let $H, a, \varepsilon, R$ be as in Lemma 3.2. Let $u$ : $\left[s_{1}, s_{2}\right] \times S^{1} \rightarrow \hat{M}$ satisfy $(u 1)$ and

$$
\max _{t \in S^{1}} r\left(s_{1}, t\right) \leq R, \quad \min _{t \in S^{1}} r\left(s_{2}, t\right) \geq 2 R
$$

Then

$$
A_{H\left(s_{2}\right)}\left(u\left(s_{2}\right)\right)-A_{H\left(s_{1}\right)}\left(u\left(s_{1}\right)\right) \geq R \varepsilon .
$$

Proof. Since the action is increasing with $s$, we can make $s_{1}$ larger and $s_{2}$ smaller until

$$
\begin{gathered}
\max _{t \in S^{1}} r\left(s_{1}, t\right)=R, \quad \min _{t \in S^{1}} r\left(s_{2}, t\right)=2 R, \\
u(s) \in Z(R) \text { for all } s \in\left[s_{1}, s_{2}\right] .
\end{gathered}
$$

Then by Lemma 3.2,

$$
\begin{aligned}
A_{H\left(s_{2}\right)}\left(u\left(s_{2}\right)\right)-A_{H\left(s_{1}\right)}\left(u\left(s_{1}\right)\right) & =\int_{s_{1}}^{s_{2}}\left\{\left\langle A_{H}^{\prime}(u), \frac{\partial u}{\partial s}\right\rangle_{L^{2}(d t)}-\int_{0}^{1} \frac{\partial H}{\partial s} d t\right\} d s \\
& \geq \int_{s_{1}}^{s_{2}} \varepsilon\left\|A_{H}^{\prime}(u)\right\|_{L^{2}(d t)} d s \\
& \geq \varepsilon\left\|\int_{s_{1}}^{s_{2}}\left|\frac{\partial u}{\partial s}\right| d s\right\|_{L^{2}(d t)} \\
& \geq\left\|r\left(s_{2}, t\right)-r\left(s_{1}, t\right)\right\|_{L^{2}(d t)} \\
& \geq \varepsilon R .
\end{aligned}
$$

\subsection{The transfer morphism}

A crucial feature of symplectic homology is the existence of a transfer morphism first observed by Viterbo [24].

We will need some homological algebra. Let $(I, \leq)$ be a directed set. A directed chain complex $\left\{C^{i}, \partial_{i}, \sigma_{i j}\right\}$ consists of a collection of chain complexes of vector spaces $\left(C^{i}, \partial_{i}\right), i \in I$, and chain maps $\sigma_{i j}: C^{i} \rightarrow C^{j}$ such that the induced maps on homology $\bar{\sigma}_{i j}: H C^{i} \rightarrow H C^{j}$ satisfy

$$
\bar{\sigma}_{j k} \circ \bar{\sigma}_{i j}=\bar{\sigma}_{i k} \text {. }
$$

A short exact sequence of directed chain complexes is a collection of chain maps $\lambda_{i}: B^{i} \rightarrow C^{i}, \pi_{i}: C^{i} \rightarrow A^{i}$ such that in the diagram

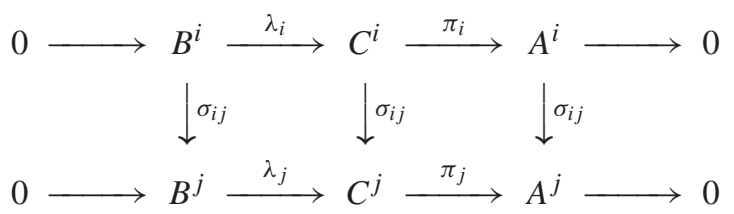


the rows are exact, and the squares commute on the level of homology. We obtain exact triangles in homology

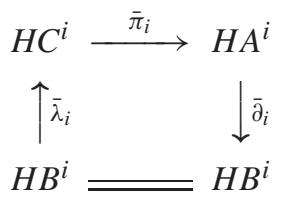

whose maps commute with the induced maps $\bar{\sigma}_{i j}$ on homology. Since exactness is preserved under direct limits, this gives rise to an exact triangle on the direct limits of homology,

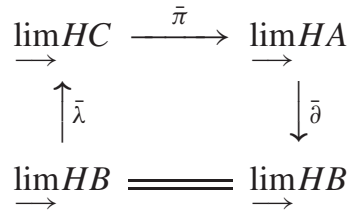

If all dimensions are finite we read off from the exact triangle that

$$
\operatorname{dim} \underset{\longrightarrow}{\lim } H C-\operatorname{dim} \underset{\longrightarrow}{\lim H A} \stackrel{\operatorname{dim}}{\underset{\lim }{\longrightarrow} H B}-2 \operatorname{dim} \operatorname{ker} \bar{\lambda}
$$

So we have proved

Lemma 3.4. Consider a short exact sequence of directed chain complexes as above. Then there exists an exact triangle

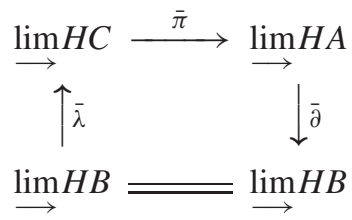

If $\operatorname{dim} \underset{\lim }{\longrightarrow} H B<\infty$ then either $\operatorname{dim} \underset{\lim }{\longrightarrow} H C=\operatorname{dim} \underset{\lim }{\longrightarrow} H A=\infty$, or both dimensions are finite and

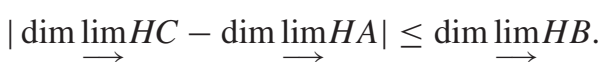

Now consider a codimension 0 submanifold $W$ of $(M, \omega)$ with $\partial W \omega$-convex, i.e. there exists a contact form $\lambda$ on $\partial W$ with $d \lambda=\left.\omega\right|_{\partial W}$. The form $\lambda$ provides a canonical way to fix the arbitrary constants $A\left(x_{\alpha}\right)$ in the definition of the action functional: We can compute symplectic homology of $W$ using Hamiltonians that are $C^{2}$-small Morse functions in the interior of $W$ and increasing sharply near $\partial W$. The 1-periodic orbits of such Hamiltonians are either constant orbits inside $W$, corresponding to critical points, or nonconstant orbits near $\partial W$. Since all such orbits are homotopic to loops in $\partial W$, this shows that $S H_{*}^{\alpha}(W, \omega)$ is nonzero only for homotopy classes $\alpha$ of loops that can be homotoped into $\partial W$. So we can choose 
the base loops $x_{\alpha}$ to lie on $\partial W$ and define their action as $A\left(x_{\alpha}\right):=\int_{x_{\alpha}} \lambda$. In particular, $A\left(x_{0}\right)=0$ for the constant base loop. With this normalisation,

$$
A_{H}(x)=\int_{x} \lambda-\int_{0}^{1} H(t, x) d t
$$

for every loop $x$ on $\partial W$

Lemma 3.5. There exists a family of symplectic forms $\omega_{i}$ on $M, \omega_{i}=\omega$ near $\partial W$ and outside $W$; a cofinal system $H_{1} \leq H_{2} \leq \ldots$ of Hamiltonians on $\left(M, \omega_{i}\right)$; and monotone homotopies $H_{i, i-1}$ between $H_{i}$ and $H_{i-1}$ with the following properties:

1. $K_{i}:=\left.H_{i}\right|_{W}$ form a cofinal system of admissible Hamiltonians on $\left(W, \omega_{i}\right)$, and $K_{i, i-1}:=\left.H_{i, i-1}\right|_{W}$ are monotone homotopies between $K_{i}$ and $K_{i-1}$;

2. all 1-periodic orbits of $H_{i}$ in $W$ have positive (Hamiltonian) action, and all 1-periodic orbits of $H_{i}$ in $M \backslash W$ have negative action;

3. all gradient trajectories of $H_{i}$ or $H_{i, i-1}$ connecting 1-periodic orbits in $W$ are entirely contained in $W$.

Proof. The first argument follows [24]. Let $(\hat{W}, \hat{\omega})$ be the completion of $(W, \omega)$. It will be convenient to use the coordinate $z=e^{r}$ rather than $r$. Then $\hat{W}=$ $W \cup(\partial W \times[1, \infty))$ and $\hat{\omega}=d(z \lambda)$ on $\partial W \times[1-\gamma, \infty)$ for some $\gamma>0$. Consider a Hamiltonian $K$ on $\hat{W}$ such that $K$ is $C^{2}$-small and $<0$ inside $W$ and $K=h(z)$ on $\partial W \times[1-\gamma, \infty)$, where $h$ is a small perturbation of the function depicted in Fig. 2 with slope $a \notin \mathcal{A}(\partial W, \lambda)$.

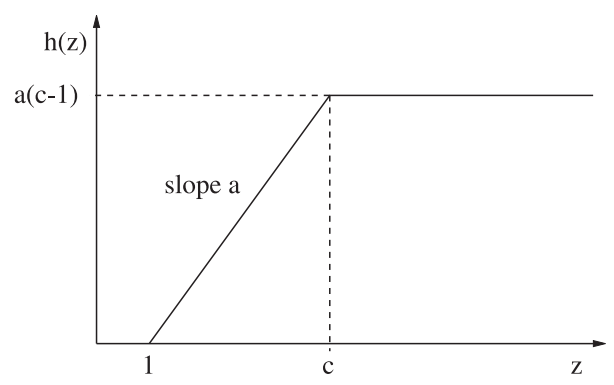

Fig. 2.

In view of the normalisation of the action functional, 1-periodic solutions at $z$ have Hamiltonian action $z h^{\prime}(z)-h(z) . K$ has 4 types of 1-periodic solutions:

- critical points in $W$ of action $>0$;

- nonconstant orbits near $z=1$ of action $\approx 1 \cdot h^{\prime}(z)>0$;

- critical points for $z \geq c$ of action $\approx-a(c-1)<0$;

- nonconstant orbits near $z=c$ of action $\approx c h^{\prime}(z)-a(c-1)<0$ for large $c$.

For the last statement, note that since $a \notin \mathcal{A}(\partial W, \lambda)$, there exists an $\eta>0$ such that $\left|h^{\prime}(z)-a\right|>\eta$ for every 1-periodic orbit. Thus $c h^{\prime}(z)-a(c-1) \leq$ $c(a-\eta)-a(c-1)=a-c \eta<0$ for $c$ sufficiently large. 
For fixed slope $a$ the maximal action difference between 1-periodic orbits in $W$ is bounded. Therefore, by Lemma 3.3, for large $c$ all gradient trajectories connecting orbits in $W$ are contained in $\{z<c\}$.

Let $\tilde{K}_{1} \leq \tilde{K}_{2} \leq \ldots$ be a cofinal sequence of such Hamiltonians on $\tilde{W}$ with slopes $a_{i} \rightarrow \infty$ and constants $c_{i} \rightarrow \infty$. Making the $c_{i}$ larger and using Lemma 3.3 again, we find monotone homotopies $\tilde{K}_{i, i-1}$ such that all gradient trajectories connecting orbits in $W$ are contained in $\left\{z<c_{i}\right\}$.

Switch back the coordinate from $z=e^{r}$ to $r$, writing $\gamma=e^{\delta}$ and $c_{i}=r^{R_{i}}$. Pick functions $f_{i}:[-\delta, 0] \rightarrow\left[-\delta, R_{i}\right]$ satisfying

- $f_{i}(r)=r$ near $-\delta$;

- $f_{i}(r)=r+R_{i}$ near 0 ;

- $f_{i}^{\prime}(r)>0$ for all $r$.

Define $\psi_{i}: W \rightarrow W \cup\left(\partial W \times\left[0, R_{i}\right]\right)$ by

$$
\begin{cases}\psi_{i}(y, r):=\left(y, f_{i}(r)\right) & \text { on } \partial W \times[-\delta, 0] \\ \psi_{i}(x):=x & \text { in } W \backslash(\partial W \times[-\delta, 0]) .\end{cases}
$$

Use $\psi_{i}$ to pull the following quantities back to $W$ :

$$
\omega_{i}:=e^{-R_{i}} \psi_{i}^{*} \hat{\omega}, \quad K_{i}:=e^{-R_{i}} \psi_{i}^{*} \tilde{K}_{i}, \quad K_{i, i-1}:=e^{-R_{i}} \psi_{i}^{*} \tilde{K}_{i, i-1} .
$$

Note that $\omega_{i}=\omega$ near $\partial M$ for all $i$, and the $K_{i}$ have slope $a_{i} \rightarrow \infty$ and value $a_{i}\left(1-e^{-R_{i}}\right) \rightarrow \infty$ near $\partial W$. Extend $K_{i}$ to an admissible Hamiltonian $H_{i}$ on $M$ by a small perturbation of the constant function $a_{i}\left(1-e^{-R_{i}}\right)$, and then increasing to slope $b_{i}$ near $\partial M$ (see [24]). The 1-periodic orbits of $H_{i}$ outside a neighbourhood of $W$ are either critical points or nonconstant orbits near $\partial M$, both of which have negative action for $b_{i}$ small compared to $a_{i}$. Since $a_{i} \rightarrow \infty$, we can choose $b_{i} \rightarrow \infty$ with this property, so the $H_{i}$ form a cofinal sequence for $\left(M, \omega_{i}\right)$ with the desired properties. Extending the monotone homotopies $K_{i, i-1}$ to $H_{i, i-1}$ on $M$ in the same way finishes the proof.

Let $H_{i}, H_{i, i-1}$ be as in Lemma 3.5. Let $\left(C^{i}, \partial_{i}, \sigma_{i j}\right)$ be the directed chain complex given by

$$
\left\{\begin{array}{c}
C^{i}:=C_{*}^{\alpha}\left(M, \omega_{i}, H_{i}\right), \\
\partial_{i}:=\partial\left(\omega_{i}, H_{i}\right): C^{i} \rightarrow C^{i}, \\
\sigma_{i j}:=\sigma\left(\omega_{i}, H_{i} ; \omega_{j}, H_{j}\right): C^{i} \rightarrow C^{j} .
\end{array}\right\}
$$

Let $B_{i} \subset C_{i}$ be the subspace generated by periodic orbits of action $<0$, and $A_{i}:=C_{i} / B_{i}$ the quotient space generated by orbits of action $\geq 0$. Since $\partial_{i}$ decreases action, it maps $B_{i}$ into itself. The map $\sigma_{i j}$ can be written as a composition of monotonicity homomorphisms $\sigma\left(H_{k, k-1}\right)$ with fixed $\omega_{k-1}$, which are action decreasing, and homomorphisms $\sigma\left(\omega_{k}, \omega_{k-1}\right)$ for fixed Hamiltonian $H_{k}$. A compactness argument shows that if the latter homotopy is sufficiently slow then it maps orbits of negative action to orbits of negative action. This shows that $\sigma_{i j}$ maps $A_{i}$ 
to $A_{j}$, and $\left(A_{i}\right)$ is a subcomplex of $\left(C_{i}\right)$. So we have a short exact sequence of directed chain complexes

$$
0 \longrightarrow B^{i} \stackrel{\lambda_{i}}{\longrightarrow} C^{i} \stackrel{\pi_{i}}{\longrightarrow} A^{i} \longrightarrow 0,
$$

where $\lambda_{i}$ is the inclusion and $\pi_{i}$ the projection. By properties 2 and 3 in Lemma 3.5, the directed chain complex $\left(A_{i}, \partial_{i}, \sigma_{i j}\right)$ is given by

$$
\left\{\begin{array}{c}
A^{i}=C_{*}^{i_{\#}^{-1} \alpha}\left(W, \omega_{i}, K_{i}\right), \\
\partial_{i}:=\partial\left(\omega_{i}, K_{i}\right): C^{i} \rightarrow C^{i}, \\
\sigma_{i j}:=\sigma\left(\omega_{i}, K_{i} ; \omega_{j}, K_{j}\right): C^{i} \rightarrow C^{j},
\end{array}\right\}
$$

where $i: W \hookrightarrow M$ is the inclusion. By the remark at the end of Sect. 3.1, since $\omega_{i}=\omega$ near $\partial W$ and $\partial M$, the direct limits of homologies are

$$
\begin{aligned}
& \underset{\lim }{\longrightarrow} C C=S H_{*}^{\alpha}(M, \omega), \\
& \stackrel{\lim }{\longrightarrow} H A=S H_{*}^{i_{\#}^{-1} \alpha}(W, \omega) .
\end{aligned}
$$

Denote $\lim H B$ by $S H_{*}^{j_{\#}^{-1} \alpha}(M, W, \omega)$, where $j: M \backslash W \hookrightarrow M$ is the inclusion. Then Lemma 3.4 yields

Proposition 3.6. Let $W$ be a codimension zero submanifold of $(M, \omega)$ with $\omega$ convex boundary $\partial W$. Then there exists an exact triangle

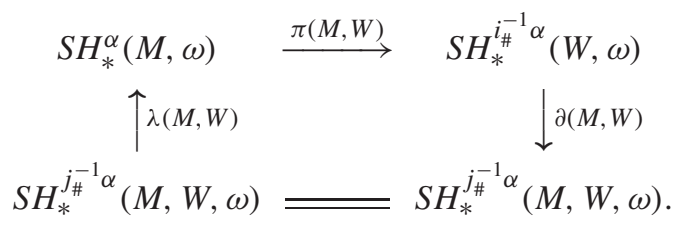

If $\operatorname{dim} S H_{*}^{j_{\#}^{-1} \alpha}(M, W, \omega)<\infty$ then either $\operatorname{dim} S H_{*}^{\alpha}(M, \omega)=\operatorname{dim} S H_{*}^{i_{\#}^{-1} \alpha}(W, \omega)$ $=\infty$, or both dimensions are finite and

$$
\left|\operatorname{dim} S H_{*}^{\alpha}(M, \omega)-\operatorname{dim} S H_{*}^{i_{\#}^{-1} \alpha}(W, \omega)\right| \leq \operatorname{dim} S H_{*}^{j_{\#}^{-1} \alpha}(M, W, \omega) .
$$

The homomorphism $\pi(M, W)$ is the dual of the transfer morphism in [24]. A first application of the transfer morphism is a proof of

\section{Lemma 3.7 (Viterbo [24]).}

1. $S H_{k}^{\alpha}(M, \omega)$ does not depend on the choice of contact form $\lambda$ on $\partial M$ with $d \lambda=\left.\omega\right|_{\partial M}$

2. $S H_{k}^{\alpha}(M, \omega)$ depends only on the homotopy class of $\omega$ in the space of symplectic forms satisfying $(\omega 1-2)$. 
Proof. (sketch) 1 . Let $\lambda_{0}, \lambda_{1}$ be two contact forms on $\partial M$ with $d \lambda_{i}=\left.\omega\right|_{\partial M}$. Then $\lambda_{t}:=(1-t) \lambda_{0}+t \lambda_{1}$ is a homotopy of contact forms satisfying $d \lambda_{t}=\left.\omega\right|_{\partial M}$. By Lemma 2.2, there exists a symplectic form $\omega_{1}$ on $M_{1}:=M \cup\left(\partial M \times\left[0, R_{1}\right]\right)$ and a nonzero expanding vector field $X_{1}$ on $\partial M \times\left[0, R_{1}\right]$ such that $\omega_{1}=\omega$ on $M$, $i_{X_{1}} \omega_{1}=e^{r} \lambda_{0}$ near $r=0$ and $i_{X_{1}} \omega_{1}=e^{r} \lambda_{1}$ near $r=R_{1}$.

In this situation the transfer morphism $\pi\left(M_{1}, M\right)$ is an isomorphism. This can be seen as follows: The same construction as above yields an extension of $\left(\omega_{1}, X_{1}\right)$ to $\left(\omega_{2}, X_{2}\right)$ on $M_{2}:=M \cup \partial M \times\left[0, R_{2}\right]$ with $i_{X_{2}} \omega_{2}=e^{r} \lambda_{0}$ near $r=R_{2}$. Then $\pi\left(M_{1}, M\right) \circ \pi\left(M_{2}, M_{1}\right)=\pi\left(M_{2}, M\right)$, and $\pi\left(M_{2}, M\right)$ induces the rescaling isomorphism $S H_{k}^{\alpha}\left(M_{2}, \omega_{2}\right) \cong S H_{k}^{\alpha}\left(M, e^{R_{2}} \omega\right) \cong S H_{k}^{\alpha}(M, \omega)$. Reversing the argument shows that $\pi\left(M_{1}, M\right)$ is an isomorphism. It follows that $S H_{k}^{\alpha}\left(M, \omega, \lambda_{0}\right) \cong S H_{k}^{\alpha}\left(M, e^{R_{1}} \omega, e^{R_{1}} \lambda_{1}\right) \cong S H_{k}^{\alpha}\left(M, \omega, \lambda_{1}\right)$.

2. Let $\left(\omega_{t}\right)_{t \in[0,1]}$ be a smooth family of symplectic forms and $\lambda_{t}$ contact forms with $d \lambda_{t}=\left.\omega_{t}\right|_{\partial M}$. Now the proof is analogous to part 1, again using Lemma 2.2.

\subsection{Handle attaching and proof of Theorem 1.11}

In this subsection we investigate how symplectic homology changes under handle attaching. In the absence of chords, this change is determined by the local Floer homology of a critical point.

Consider a monotone homotopy $H(s, t, x)$ between admissible Hamiltonians $H_{-} \geq H_{+}$on $(\hat{M}, \hat{\omega})$ such that $H(s)$ is regular except for finitely many $s$. Suppose that there exists a point $p \in M$ which is a critical point for all $H(s)$ and such that

$$
A_{H(s)}(p)<A_{H(s)}(x)
$$

for all 1-periodic orbits $x \neq p$ of $H(s)$, for all $s$. Assume also that $H(s, t, p)$ is constant in $t$ for all $s$. Write the point $p$ as $p_{ \pm}, p_{s}$ when viewed as a 1-periodic orbit of $H_{ \pm}, H(s)$. The point $p$ can be connected by a path $\bar{p}$ of constant loops to the base point of the trivial homotopy class $\alpha=0$ of loops. With this path $\bar{p}$ understood, we can view $p_{ \pm}$as elements in $\mathcal{P}^{0}\left(H_{ \pm}\right)$.

Lemma 3.8. For $H$ and $p$ as above the following identities in the Floer complex hold:

1. $\partial\left(H_{ \pm}\right) p_{ \pm}=0$.

2. $\left\langle\sigma\left(H_{+}, H_{-}\right) p_{+}, x_{-}\right\rangle=0$ for all $p_{-} \neq x_{-} \in \mathcal{P}^{0}\left(H_{-}\right)$.

3. If $p$ is nondegenerate for all $H(s)$ then $\sigma\left(H_{+}, H_{-}\right) p_{+}=p_{-}$.

4. If $\operatorname{ind}_{C Z}\left(p_{-}\right) \neq \operatorname{ind}_{C Z}\left(p_{+}\right)$then $\sigma\left(H_{+}, H_{-}\right) p_{+}=0$.

Proof. (1) holds because $\partial\left(H_{ \pm}\right)$decreases the Hamiltonian action.

If (2) does not hold, then there exist arbitrarily close $s_{1}<s_{2}$ such that $\left\langle\sigma\left(H_{s_{2}}, H_{s_{1}}\right) p_{s_{2}}, x_{1}\right\rangle \neq 0$ for some $p_{s_{1}} \neq x_{1} \in \mathcal{P}^{0}\left(H_{s_{1}}\right)$. But for $\left|s_{2}-s_{1}\right|$ small, $A_{H_{s_{2}}}(p)<A_{H_{s_{1}}}\left(x_{1}\right)$ for all $p_{1} \neq x_{1} \in \mathcal{P}^{0}\left(H_{s_{1}}\right)$, which yields a contradiction because $\sigma\left(H_{s_{2}}, H_{s_{1}}\right)$ decreases action.

For (3), fix a contractible neighbourhood $U$ of $p$. For $s_{1}<s_{2}$ denote by $H_{\left[s_{1}, s_{2}\right]}$ a monotone homotopy between $H_{s_{2}}$ and $H_{s_{1}}$ obained from $H$ by rescaling in $s$. 
A compactness argument as in [8] shows that for $\left|s_{2}-s_{1}\right|$ sufficiently small each $u \in \mathcal{M}\left(p_{s_{1}}, p_{s_{2}} ; H_{\left[s_{1}, s_{2}\right]}\right)$ is contained entirely in $U$. Then an implicit function type argument as in [8], based on the nondegeneracy of $p$, shows that for $\left|s_{2}-s_{1}\right|$ sufficiently small the only $u \in \mathcal{M}\left(p_{s_{1}}, p_{s_{2}} ; H_{\left[s_{1}, s_{2}\right]}\right)$ is the constant map $u(s, t) \equiv p$. Hence $\sigma\left(H_{s_{2}}, H_{s_{1}}\right) p_{s_{2}}=p_{s_{1}}$, and since $\sigma\left(H_{+}, H_{-}\right)$is a composition of such maps, (3) follows.

For (4), let $s^{*}$ be a value at which $p$ is singular for $H\left(s^{*}\right)$ and changes ConleyZehnder index. Let $s_{1}<s^{*}<s_{2}$ be values for which $p$ is regular such that $\operatorname{ind}_{C Z}\left(p_{s_{1}}\right) \neq \operatorname{ind}_{C Z}\left(p_{s_{2}}\right)$. For $\left|s_{2}-s_{1}\right|$ small we can modify the monotone homotopy $H$ such that $H\left(s_{1}, t, p\right)=H\left(s_{2}, t, p\right)$ for all $t$. Then $A_{H\left(s_{1}\right)}(p)=A_{H\left(s_{2}\right)}(p)$, and since $\sigma\left(H_{s_{2}}, H_{s_{1}}\right)$ decreases action, the only $u \in \mathcal{M}\left(\left[p_{s_{1}}, \bar{p}_{1}\right],\left[p_{s_{2}}, \bar{p}_{2}\right]\right.$; $\left.H_{\left[s_{1}, s_{2}\right]}\right)$ is the constant map $u(s, t) \equiv p$. Here $\bar{p}_{i}$ are not necessarily constant paths from $p$ to the base point for the trivial homotopy class. The existence of such a $u$ implies $\left[p_{s_{1}}, \bar{p}_{1}\right]=\left[p_{s_{1}}, \bar{p}_{2}\right]$. Hence

$$
\operatorname{ind}_{C Z}\left(\left[p_{s_{1}}, \bar{p}_{1}\right]\right)=\operatorname{ind}_{C Z}\left(\left[p_{s_{1}}, \bar{p}_{2}\right]\right) \neq \operatorname{ind}_{C Z}\left(\left[p_{s_{2}}, \bar{p}_{2}\right]\right) \text {. }
$$

This proves that

$$
\left\langle\sigma\left(H_{s_{2}}, H_{s_{1}}\right)\left[p_{s_{2}}, \bar{p}_{2}\right],\left[p_{s_{1}}, \bar{p}_{1}\right]\right\rangle=0,
$$

from which (4) follows.

Proof of Theorem 1.11. The proof consists in the computation of the relative homology $S H_{*}^{j_{\#}^{-1} \alpha}(M, W, \omega)$ as defined in Proposition 3.6. Let $\omega_{i}, H_{i}, K_{i}, H_{i, i-1}$, $K_{i, i-1}$ be as in Lemma 3.5. In this situation the definition of $H_{i}$ can be modified as follows: Instead of making $K_{i}$ flat near $\partial W$ and extending it as a constant over $M$, we can retain its slope $a_{i}$ near $\partial W$ and extend it over the handle by a function $\psi$ as in Lemma 2.5. The resulting Hamiltonian $H_{i}$ on $M$ has slope $b_{i}>a_{i}$ near $\partial M$, and its only 1-periodic orbit on the handle is the critical point $p_{i}$ in its center.

If $L$ possesses no chord then by choosing the handle sufficiently thin we can avoid creating new 1-periodic orbits that traverse the handle. The same can be achieved for $k<n$ by a perturbation of $L$ (see [24]). In either case, the only 1-periodic orbit of $H_{i}$ on $M \backslash W$ is the critical point $p_{i}$.

Define the monotone homotopies in a similar way such that the $\omega_{i}, H_{i}, H_{i, i-1}$ satisfy the properties in Proposition 3.6. With these choices, the chain groups $B^{i}$ defined preceding Proposition 3.6 are generated by the single elements $p_{i}$. Since the $p_{i}$ have lower action than all other 1-periodic orbits of $H_{i}$, we are precisely in the situation of Lemma 3.8.

(3) follows immediately from Proposition 3.6 because $\operatorname{dim} S H_{*}^{j_{\#}^{-1} \alpha}(M, W, \omega) \leq 1$.

(2) holds because $S H_{*}^{j_{\#}^{-1} \alpha}(M, W, \omega)$ is generated by the critical point on the handle which lies in the trivial homotopy class of loops in $M$.

(1) For $k<n$ we can always increase the Conley-Zehnder index of $p_{i}$ by multiples of 2 by increasing the second derivative of $H_{i}$ at $p_{i}$. So we can achieve ind $C Z\left(p_{i}\right)>$ $\operatorname{ind}_{C Z}\left(p_{i-1}\right)$ for all $i$. Moreover, the monotone homotopies $H_{i, i-1}$ can be chosen to satisfy the assumptions of Lemma 3.8 near the critical point $p_{i}=p_{i-1}$. It 
follows that $\sigma\left(H_{i-1}, H_{i}\right) p_{i-1}=0$ for all $i$. So $p_{i}$ dies in the direct limit, hence $S H_{*}^{j_{\#}^{-1} \alpha}(M, W, \omega)=0$, and the statement follows from Proposition 3.6.

Acknowledgements. I thank T. Ekholm, Y. Eliashberg, J. Etnyre and H. Hofer for many fruitful discussions; R. Gompf for the Kirby diagrams; and S. Kim who, perhaps unknowingly, initiated this paper.

\section{References}

1. C. Abbas: A note on V.I. Arnold's Chord Conjecture. Int. Math. Res. Notices 4, 217-222 (1999)

2. C. Abbas: Finite energy surfaces and the Chord Problem. Duke Math. J. 96, 241-316 (1999)

3. V.I. Arnold: First steps in symplectic topology. Russ. Math. Surv. 41, 1-21 (1986)

4. M. Audin, J. Lafontaine: Holomorphic Curves in Symplectic Geometry. Birkhäuser Progress Math. 117 (1994)

5. S. Bolotin: Libration motions of natural dynamical systems. (Russian) Vestnik Moskov. Univ. Ser. I Mat. Mekh. 6, 72-77 (1978)

6. K. Cieliebak: A geometric obstruction to the contact type property. Math. Z. 228, 451-487 (1998)

7. K. Cieliebak, A. Floer, H. Hofer: Symplectic homology II: A general construction. Math. Z. 218, 103-122 (1995)

8. K. Cieliebak, A. Floer, H. Hofer, K. Wysocki: Applications of symplectic homology II: Stability of the action spectrum. Math. Z. 223, 27-45 (1996)

9. C. Conley, E. Zehnder: Morse type index theory for flows and periodic solutions of Hamiltonian equations. Commun. Pure Appl. Math. 37, 207-253 (1984)

10. Y. Eliashberg: Topological characterization of Stein manifolds of dimension $>2$. Int. J. Math. 1, 29-46 (1990)

11. Y. Eliashberg: Symplectic geometry of plurisubharmonic functions. Lecture Notes by M. Abreu (1996)

12. Y. Eliashberg: Invariants in contact topology. Doc. Math. Extra Volume ICM 1998, $1-12(1998)$

13. Y. Eliashberg, M. Fraser: Classification of topologically trivial Legendrian knots. Geometry, topology, and dynamics (Montreal, PQ, 1995), 17-51, CRM Proc. Lecture Notes, 15. Providence, RI: Amer. Math. Soc. 1998

14. Y. Eliashberg, M. Gromov: Convex symplectic manifolds. Proc. Symp. Pure Math. 52, Part 2, 135-162 (1991)

15. A. Floer, H. Hofer: Symplectic homology I: Open sets in $\mathbb{C}^{n}$. Math. Z. 215, 37-88 (1994)

16. A. Floer, H. Hofer, K. Wysocki: Applications of symplectic homology I. Math. Z. 217, 577-606 (1994)

17. A. Givental: The nonlinear Maslov index. In: Geometry of Low-Dimensional manifolds, S. Donaldson, C. Thomas, eds., LMS Lecture Note Series vol. 151, pp. 35-43. Cambridge: Cambridge University Press 1990

18. A. Givental: Nonlinear generalization of the Maslov index. Adv. Sov. Math. 1, 71-103 (1990)

19. R. Gompf: Handlebody construction of Stein surfaces. Ann. Math. 148, 619-693 (1998)

20. R. Gompf, A. Stipsicz: 4-manifolds and Kirby calculus. Graduate Studies in Mathematics, 20. Providence, RI: Amer. Math. Soc. 1999

21. K. Mohnke: Holomorphic disks and the Chord Conjecture. Ann. Math. (to appear)

22. D. Salamon, E. Zehnder: Morse theory for periodic solutions of Hamiltonian systems and the Maslov index. Commun. Pure Appl. Math. 45, 1303-1360 (1992) 
23. D. Sullivan: Differential forms and the topology of manifolds, Proc. Int. Conf. on Manifolds and Related Topics in Topology, Tokyo 1973, Univ. of Tokyo Press, pp. 3749 (1975)

24. C. Viterbo: Functors and computations in Floer homology with applications, Part I. Geom. Funct. Anal. 9(5), 985-1033 (1999)

25. C. Viterbo: Functors and computations in Floer homology with applications, Part II. Geom. Funct. Anal. (to appear)

26. J. Weber: $J$-holomorphic curves in cotangent bundles and the heat flow. $\mathrm{PhD}$ thesis Tech. Univ. Berlin (1999)

27. A. Weinstein: Contact surgery and symplectic handlebodies. Hokk. Math. J. 20, 241$251(1991)$ 